\title{
Capsular Polysaccharide is a Main Component of Mycoplasma ovipneumoniae in the Pathogen-Induced Toll-Like Receptor-Mediated Inflammatory Responses in Sheep Airway Epithelial Cells
}

\author{
Zhongjia Jiang, ${ }^{1,2}$ Fuyang Song, ${ }^{1,2}$ Yanan Li, ${ }^{1,2}$ Di Xue, ${ }^{1,2}$ Guangcun Deng, ${ }^{1,2}$ Min Li, ${ }^{1,2}$ \\ Xiaoming Liu, ${ }^{1,2,3}$ and Yujiong Wang ${ }^{1,2}$ \\ ${ }^{1}$ Key Laboratory of Ministry of Education for Conservation and Utilization of Special Biological Resources in Western China, Ningxia \\ University, Ningxia 750021, China \\ ${ }^{2}$ College of Life Science, Ningxia University, Yinchuan, Ningxia 750021, China \\ ${ }^{3}$ Institute for Stem Cell Research, General Hospital of Ningxia Medical University, Yinchuan, Ningxia 750004, China
}

Correspondence should be addressed to Xiaoming Liu; erc1080@163.com and Yujiong Wang; wyj@nxu.edu.cn

Received 20 December 2016; Accepted 27 March 2017; Published 3 May 2017

Academic Editor: Giuseppe Valacchi

Copyright ( 2017 Zhongjia Jiang et al. This is an open access article distributed under the Creative Commons Attribution License, which permits unrestricted use, distribution, and reproduction in any medium, provided the original work is properly cited.

\begin{abstract}
Mycoplasma ovipneumoniae (M. ovipneumoniae) is characterized as an etiological agent of primary atypical pneumonia that specifically infects sheep and goat. In an attempt to better understand the pathogen-host interaction between the invading $M$. ovipneumoniae and airway epithelial cells, we investigated the host inflammatory responses against capsular polysaccharide (designated as CPS) of M. ovipneumoniae using sheep bronchial epithelial cells cultured in an air-liquid interface (ALI) model. Results showed that CPS derived from M. ovipneumoniae could activate toll-like receptor- (TLR-) mediated inflammatory responses, along with an elevated expression of nuclear factor kappa B (NF- $\kappa \mathrm{B})$, activator protein-1 (AP-1), and interferon regulatory factor 3 (IRF3) as well as various inflammatory-associated mediators, representatively including proinflammatory cytokines, such as IL1 $\beta$, TNF $\alpha$, and IL8, and anti-inflammatory cytokines such as IL10 and TGF $\beta$ of TLR signaling cascade. Mechanistically, the CPS-induced inflammation was TLR initiated and was mediated by activations of both MyD88-dependent and MyD88-independent signaling pathways. Of importance, a blockage of CPS with specific antibody led a significant reduction of $M$. ovipneumoniae-induced inflammatory responses in sheep bronchial epithelial cells. These results suggested that CPS is a key virulent component of M. ovipneumoniae, which may play a crucial role in the inflammatory response induced by M. ovipneumoniae infections.
\end{abstract}

\section{Introduction}

Mycoplasma bacteria have been extensively studied as one class of the smallest, free-living, and self-replicating bacterial cells [1]. Pathogenically, they have an ability to colonize the respiratory epithelium by attaching to the cilia of epithelial cells therefore resulting in clumping and loss of cilia and the subsequent decrease in mucociliary apparatus function [2-5]. Although attachment is of critical in the pathogenic process by causing a physical damage, it is insufficient to explain all aspects during mycoplasma infection. Numerous factors appear to act in synergy for the occurrence and development of disease during an infection of mycoplasma bacteria. For instance, immunopathological lesions associated with Mycoplasma pneumoniae are characterized by an infiltration of the perivascular and peribronchiolar spaces with lymphocytes and macrophages, suggesting an involvement of immunomodulatory effects in the host respiratory immune system [6-9].

Mycoplasma ovipneumoniae (M. ovipneumoniae) is an etiological pathogen that colonizes respiratory epithelial cells of sheep exposed to contaminated air and is known to be a 
major contributing factor to the occurrence of primary atypical pneumonia [2, 3, 10-13]. Polysaccharides, polymers of repeated mono- or di-oligosaccharide residues joined together by glycosidic bonds, are main component of the outer capsule of $M$. ovipneumoniae which play significant roles in its growth and development [14-16]. Capsular polysaccharide (designated as CPS) is cell-surface polysaccharide that is one of the few identified virulent determinants and considered to mediate interactions with host cells. It has been shown to possess various biological activities such as functions in cellular adherence, invasion, virulence, and immune modulation [17]. These functions are considerably contributing to the pathogenic mechanism of M. ovipneumoniae. Recent studies have shown that CPS of Mycoplasma pneumoniae could be recognized and subsequently combined with specific receptors on dendritic cells and macrophages, with the purpose of enhancing their viabilities against pathogenic microorganisms by promoting the secretion and expression of proinflammatory mediators, such as interleukin 10 (IL10) and IL12 [18]. However, limited information concerning its immunomodulating activities and molecular pathogenesis is currently available during M. ovipneumoniae infection. As a consequence, it is of meaning to explore the proinflammatory effects and precise molecular mechanisms of the capsular polysaccharides from $M$. ovipneumoniae.

Airway epithelium has recently been recognized as the first line of defense against $M$. pneumoniae infection, which is responsible for initiating an innate immune response by producing various array inflammatory mediators thus mediating lung inflammation $[15,19,20]$. Additionally, it plays a key role in regulating adaptive immune responses via expressing pattern recognition receptors such as toll-like receptors (TLRs) to trigger host defense responses, by interacting with dendritic cells to regulate antigen sensitization and by releasing cytokines and chemokines to recruit effector cells $[19,21]$. Therefore, airway epithelial cells serve as a bridge between innate and adaptive immunity via acting as initiators, mediators, and regulators. These evidences of roles of airway epithelium cells as immune mediators and effectors clearly indicate their involvement in inflammatory responses upon M. ovipneumoniae infection.

However, most of previous colonization models of $M$. ovipneumoniae infection employed with conventional monolayer culture of epithelial cells, rather than the multilayered and differentiated pseudostratified cells with polarized cilia and mucus secretion, are limited in their ability to accurately display the functions of ciliated cells [22-24]; therefore, they might not fully reflect the nature immune responses of respiratory epithelial cells in response to the mycoplasma infection. One the other hand, the air-liquid interface (ALI) culture models generated with primary airway epithelial cells of various species including human [25], bovine [26], and sheep [27] have been developed and used in studies of physiology and biological functions, which allows an improved mimicking of pathogen-host interactions occurring in vitro. Therefore, in the present study, the molecular mechanism of proinflammatory responses in bronchial epithelial cells of Tan sheep to M. ovipneumoniae-derived CPS was investigated using an ALI model.

\section{Materials and Methods}

2.1. Ethic Statement. The present study was approved by the ethics committee for the care and use of animals at Ningxia University.

\subsection{Propagation of M. Ovipneumoniae and Purification of} CPS. The M. ovipneumoniae Queensland Strain Y98 was cultured and propagated in a mycoplasma broth base CM403 culture media, supplemented with supplement-G SR59 (OXOID, Hampshire, UK), and $0.002 \%$ phenol red as well as an additional $10 \%$ glucose so as to maximize the production of polysaccharide. The preparation of M. ovipneumoniae CPS was carried out according to the method of Allen et al. with slight modification [28]. Briefly, the cell pellets were treated with phenol water at $60^{\circ} \mathrm{C}$ by a constant stirring for $30 \mathrm{~min}$ to remove lipophilic substances and other compounds with low molecular weight and then centrifuged at $5000 \times \mathrm{g}$ for $30 \mathrm{~min}$ at room temperature. The upper aqueous phase was then collected and dialyzed using a cellulose ester tubular membrane (exclusion limit $3500 \mathrm{Da}$ ), followed by treatment with DNase I, RNase, and pronase K. Afterwards, the resulting solution was sequentially subjected to extract with phenol/chloroform $(1: 1)$ and chloroform using a Phase-lock gel (Tiangen, Beijing, China) in order to remove residual lipophilic components of cytoplasmic membranes. The final crude polysaccharide extract (designated as CPS) was obtained by precipitation with 4.0 -fold volume of cold ethanol containing $3 \mathrm{M}$ sodium acetate at $4^{\circ} \mathrm{C}$ overnight. Above crude CPS was dissolved in distilled water and fractionated for purification using a DEAE-cellulose anionexchange chromatography column $(1.0 \mathrm{~cm} \times 5.0 \mathrm{~cm} ; \mathrm{GE}$ Healthcare Bio-Science AB, Uppsala, Sweden) equilibrated with distilled water. Polysaccharides were eluted with distilled water and $\mathrm{NaCl}$ gradient (from $0-1.0 \mathrm{M}$ ) at a flow rate of $0.5 \mathrm{~mL} / \mathrm{min}$. The eluent was then collected using an automatic collector $(300 \mu \mathrm{L} /$ tube $)$ and detected by an improved phenol-sulfuric acid method at $490 \mathrm{~nm}$. The elution peaks were finally evaluated, and the main fraction was collected as purified CPS, which was stored at $-80^{\circ} \mathrm{C}$ after lyophilization. As the preferable effect of CPS on cell viability was optimized in our previous studies, $100 \mathrm{ng} / \mathrm{mL}$ of CPS for $48 \mathrm{~h}$ was used in the following experiments.

\subsection{Production of Polyclonal Antibodies to CPS of $M$.} Ovipneumoniae. Polyclonal antiserum against purified CPS was raised in two female-specific pathogen-free New Zealand white rabbits $(1.5-2.0 \mathrm{~kg})$. Considering the low immunogenicity of pure polysaccharide, above purified CPS was first coupled with a carrier protein bovine serum albumin (BSA) to improve its immunogenicity. The rabbits were firstly injected subcutaneously with $1 \mathrm{~mL}$ of CPS-BSA-conjugated antigen solution emulsified in Freund's complete adjuvant (Sigma, St. Louis, MO, USA) and then boosted with another equivalent solution in Freund's incomplete adjuvant (Sigma, St. Louis, MO, USA) for three times with two weeks interval. Seven (7) days after the final boost, blood was droved and allowed to clot overnight and then centrifuged and the serum was subsequently decanted off and stored at $-20^{\circ} \mathrm{C}$. 
Afterwards, the polyclonal antibody against CPS was purified as IgG fraction from the resulted antiserum by affinity chromatography on a HiTrap Protein G column (GE Healthcare). The evaluation of antibody binding to CPS was eventually conducted using an indirect enzyme-linked immunosorbent assay (ELISA), as described previously [29].

2.4. Immunofluorescence Staining. M. ovipneumoniae was cultivated until the late stationary growth phase, and the sample was then pelleted by centrifugation for $30 \mathrm{~min}$ at $12000 \times \mathrm{g}$. After washing three times for $10 \mathrm{~min}$ with phosphate-buffered saline ( $\mathrm{PBS}, \mathrm{pH}=7.4$ ), bacterial cells were fixed with $4 \%$ paraformaldehyde for $15 \mathrm{~min}$ followed by a permeabilization with $0.3 \%$ TritonX-100 for $10 \mathrm{~min}$ at room temperature. Nonspecific antibody binding was subsequently blocked using 3\% normal BSA in PBS for $2 \mathrm{~h}$ at room temperature, after which rabbit anti-M. ovipneumoniae CPS IgG (self-made antibodies) $(1: 100)$ and rabbit nonspecific IgG (Beyotime, Shanghai, China) were applied and incubated in a humidified chamber at $4^{\circ} \mathrm{C}$ overnight. The binding of primary antibody was then detected by an addition of Rhodamine- (TRITC-) labeled goat-anti-rabbit IgG secondary antibody (Proteintech Group, Chicago, USA) (1:100). After extensively washing for $3 \times 5 \mathrm{~min}$, bacterial cells were mounted on slides and photographed using LEICA TCS SP2 A0BS Confocal system. Images were processed by Leica Confocal Software v.2.6.1 (Leica, Mannheim, Germany).

2.5. In Vitro ALI Culture of Sheep Bronchial Epithelium and Infection of M. Ovipneumoniae. ALI cultures of sheep bronchial epithelial cells were generated as previously described [27, 30]. Briefly, bronchial epithelial cells were obtained and seeded in polycarbonate/polyester porous collagen-precoated membranes (PCF Millicell inserts, Millipore, Bedford, MA) using Bronchial Epithelial Cell Growth Medium (BEGM) (Lonza, Basel, Switzerland) containing $5 \%$ fetal bovine serum (FBS) and cultured at $37^{\circ} \mathrm{C}$ for approximately $24-48 \mathrm{~h}$. The BEGM was subsequently removed, and a $2 \%$ Ultroser G medium (Pall, NY, USA) was applied to the basolateral side of the chamber to establish an air-liquid interface (ALI) culture system. Followed by 4-6 weeks of cultivation, epithelial cells were well polarized and highly differentiated were capable of making significant infections. For a $2.4 \mathrm{~cm}$ diameter Millicell insert membrane, normally $1 \times 10^{7}$ well-differentiated epithelial cells were determined [31]. For infection or treatment, M. ovipneumoniae cells or CPS were suspended and diluted in $2 \%$ Ultroser G medium and applied on the apical surface of ALI epithelial cells with indicated time periods until the cells and cultures were harvested for analysis. In addition, an equal volume of $2 \%$ Ultroser $G$ medium was used as an untreated control. Normally, volumes of $0.5 \mathrm{~mL}$ and $1 \mathrm{~mL}$ were employed for coving the wells with diameters of $1.2 \mathrm{~cm}$ and $2.4 \mathrm{~cm}$, respectively.

2.6. RNA Isolation and Quantitative Real-Time PCR (RT$P C R)$. Total cellular RNA was extracted using the TRIzol Reagent (Invitrogen, Carlsbad, CA, USA) according to the manufacturer's instructions. The quality and quantity of RNA were ascertained by a NanoDrop system (Thermo Fisher Scientific, Waltham, MA, USA). First-strand complementary DNA (cDNA) was synthesized with the PrimeScript $^{\mathrm{TM}}$ RT Master Mix Reagent Kit (TaKaRa, Tokyo, Japan) using $10 \mu \mathrm{L}$ of reaction mixture containing $500 \mathrm{ng}$ of total RNA, $2 \mu \mathrm{L}$ of $5 \mathrm{x}$ PrimeScript reaction mix, and RNase-free water. The RT-PCR was performed at $37^{\circ} \mathrm{C}$ for $15 \mathrm{~min}$ and was stopped by heating to $85^{\circ} \mathrm{C}$ for $5 \mathrm{sec}$. The primers specific for the genes of interest were designed and synthesized in Sangon Biotech Inc. (Shanghai, China) using bioinformatic tools and are listed in Table 1. The quantitative real-time PCR (qRT-PCR) was performed using TransStart ${ }^{\circledR}$ Tip Green qPCR SuperMix (2x) (TransGen, Beijing, China) as follows: a precycling stage at $94^{\circ} \mathrm{C}$ for $30 \mathrm{sec}$, then 40 cycles of denaturation at $94^{\circ} \mathrm{C}$ for $5 \mathrm{sec}$, and an annealing step at $60^{\circ} \mathrm{C}$ for $30 \mathrm{sec}$. In a parallel experiment, each sample was tested three times and $\beta$-actin was used as an internal control. Fold changes over controls were calculated by a $2^{-\Delta \Delta C T}$ method to determine mRNA expression levels [32].

2.7. Immunoblotting Analysis. 6-well ALI inserts were infected with $M$. ovipneumoniae cells or treated with CPS as indicated conditions for $48 \mathrm{~h}$. After extensively washing for $3 \times 1 \mathrm{~min}$, cells were lysed using the Whole Protein Extraction Kit (KeyGEN, Nanjing, China) according to the manufacturer's instructions and harvested by centrifugation at $12,000 \times \mathrm{g}$ for $5 \mathrm{~min}$ at $4^{\circ} \mathrm{C}$. Protein concentrations were determined by the BCA Assay kit (KeyGEN, Nanjing, China). $60 \mu \mathrm{g}$ of total protein of each lysate were then resolved by $8-12 \%$ SDS-PAGE and subsequently transferred to polyvinylidene fluoride (PVDF) membranes (Millipore, Bedford, MA, USA). Afterwards, membranes were blocked with 5\% nonfat milk in TBST at room temperature for 2$4 \mathrm{~h}$, followed by incubating with antibodies against protein of interest overnight at $4^{\circ} \mathrm{C}$. Antibodies used in this study included rabbit anti-Toll-like receptor 4, rabbit anti-AP-1, rabbit anti-p-AP-1 (Cell Signaling Technology, Beverly, MA, USA), rabbit anti-MyD88, rabbit anti-IRAK1, rabbit anti-IRAK4, rabbit anti-TRAF6, rabbit anti-TAB1, rabbit anti-TRIF, rabbit anti-TRAF3, rabbit anti-IRF3, rabbit antiIRF5, rabbit anti- $\beta$-actin (Proteintech Group, Chicago, USA), rabbit anti-IRAK2, rabbit anti-TBK1 (ABGENT, San Diego, USA), and rabbit anti-p-NFkB (Signalway Antibody, Maryland, USA). After washing with TBST for $3 \times 10 \mathrm{~min}$, membranes were striped with appropriate HRP-conjugated secondary antibodies for 1-2 $\mathrm{h}$ at room temperature, followed by visualizing using the enhanced Western Bright ECL reagents (Advansta, Menlo Park, CA, USA). Eventually, bands were imaged and densimetrically analyzed and semiquantified by Image J software version 1.46 (NIH, Bethesda, MD, USA), as previously described [33].

2.8. ELISA for Cytokine Measurements. Commercial enzymelinked immunosorbent assay (ELISA) kits obtained from Enzyme-Linked Biotechnology (Shanghai, China) were used for the measurement of TNF $\alpha$, IL1 $\beta$, IL6, IL8, and IL10 protein levels in culture supernatant. The 4-week ALI cultures were infected with $M$. ovipneumoniae (multiplicity of infection, MOI $=30)$ or treated with CPS $(100 \mathrm{ng} / \mathrm{mL})$ for $48 \mathrm{~h}$, 
TABLE 1: Sheep gene specific primers for qRT-PCR.

\begin{tabular}{|c|c|c|}
\hline Gene & Forward (primer sequence $5^{\prime}-3$ ) & Forward (primer sequence $5^{\prime}-3^{\prime}$ ) \\
\hline$\beta$-actin & GCTCTCTTCCAGCСТTCСТT & AGGTCTTTGCGGATGTCG \\
\hline TLR1 & CAGTGGGATTCAGTAGGT & TTGTTTCCAGGGATAAGT \\
\hline TLR2 & TCCTGTTGCTCCTGCTCAC & CTTCCTGGGCTTCCTCTTG \\
\hline TLR4 & TTTGTCCCTGAACCCTTTAGAA & AAACCAGCCAGACCTTGAATAC \\
\hline TLR6 & CAGTGAATAGTTTAGGGTGCTTACA & CGTTGGTCTTCCAGTGAGTC \\
\hline MyD88 & ATGGTGGTGGTTGTCTCTGAC & GGAACTCTTTCTTCATTGGCTTGT \\
\hline IRAK1 & TGGACACCGACACCTTCAG & CTGCСТССТССТСААССАAG \\
\hline IRAK2 & TATGGCTGGATGAAGAACCA & CAGGGAGGGAGAATGAATGA \\
\hline IRAK4 & TGTGCTACCTGACTCCTCAAG & АСТССАААСССТССТТСТСС \\
\hline TRAF6 & TCAGAGAACAGATGCCCAAT & GCGTGCCAAGTGATTCCT \\
\hline TAB1 & GGGTCTTCAATGGCTACGAT & TCCAACGCCTTGAGTCTGT \\
\hline TRAF3 & CCCTGGAGAAGAAGGTTTCC & CGATGACTCGCTGTAAATGG \\
\hline TBK1 & TGTATCTCCGACTGCCTGCT & GGTTCTGCTCTCCACTCACAG \\
\hline TRAM1 & CTGCCACAGAAGAACAAG & AAGCCCTCCACAAGATAG \\
\hline TRAM2 & CGTTAGTGTCCCTTATCGTG & CGTCAGTTCATCAGCACCT \\
\hline IRF5 & GCACCСТАТTCTTTACССАA & TCAGGCAGGAGTTGTTCG \\
\hline $\mathrm{NF}-\kappa \mathrm{B}$ & CGAGGATGATGAGAATGG & CAGGAACACGGTTACAGG \\
\hline IRF3 & GAAGGAAGTGTTGCGTTTAGC & TGTCTGCCATTGTCTTGAGC \\
\hline IL $1 \alpha$ & CCCTGGATACCTCGGAAAC & AAACTCAACCGTCTCTTCTTCA \\
\hline $\operatorname{IL} 1 \beta$ & TCTCССТAAAGAAGCCATAC & AGCGTCTCAGCACGAATA \\
\hline IL4 & CGTAAGAAGGGCGAGCAG & AGGTGGAGGTAGGGTCAGC \\
\hline IL6 & TTGAGGGAAATCAGGAAACTGT & TGTGGCTGGAGTGGTTATTAGA \\
\hline IL8 & GCTGGCTGTTGCTCTCTTG & GTGGAAAGGTGTGGAATGTGTT \\
\hline IL10 & TTTCCCTGACTGCCСТCTAAT & СTCCCTGGTTTCTCTTC \\
\hline IL12A & GGGCATTGTCTGTCTTCT & TCTTCCAGGGAGGGTTTC \\
\hline IL12B & ATTCTCGGCAGGTGGAAGT & CTGAGGTTTGGTCTGTGAAGAG \\
\hline IL13 & CCTATGCGTCTGCTTCTC & ATCCTCTTGGTCCTGTGG \\
\hline IL17A & AGCACAAGCCCATCCATC & CCTGCCTTCACAAGAGCC \\
\hline IL17B & TGGTCGGCAGACTAAGAT & CAAGAATACTGGAGTGGGTT \\
\hline IL18 & AAATGGCGAAGACCTGGAATC & TCCCTGGCTAATGAAGAGAACTT \\
\hline IL23A & AACCAGACGCACAGAACA & AGCAGCAGCATCACAACT \\
\hline IL33 & AGGGCTTCACCTTGGGTAATA & CACCTTGTCTTTCTCTTGGTCTC \\
\hline TNF $\alpha$ & СССАACTCССТCTGTTTATGTT & GGACACCTTGACCTCCTGAATA \\
\hline $\operatorname{IFN} \beta$ & AGAACCTCCTGTGGCAGTTAC & CAATACGGCATCTTCCTTCC \\
\hline TGF $\beta$ & GTTCTTCAACACGTCCGAG & CACGTGCTGCTCCACTTTTA \\
\hline
\end{tabular}

prior to the collection of the supernatant and measurement of inflammation-associated factor concentrations. Absorbance was eventually measured at $450 \mathrm{~nm}$ using a microplate reader (Thermo Fisher Scientific, Waltham, MA, USA) according to the manufacturer's protocols, and standard curves were used for ascertaining cytokine concentration.

2.9. Statistical Analysis. All data in this study were presented as the mean $\pm \mathrm{SD}$ of data from at least three independent experiments. SPSS statistics 17.0 (SPSS Inc., Chicago, IL, USA) was used for the statistical analysis. Statistical differences between groups were performed using one-way analysis of variance (ANOVA) followed by post hoc Tukey's test. $P$ values $<0.05$ were considered statistically significant.

\section{Results}

3.1. Validation of CPS Antibody Using Immunofluorescence Staining. In order to assess the immunogenic potential of CPS and be able to correlate further experimental results, it is of importance to apply reliable laboratory test methods that provide valid assessments of antibody responses. To this end, immunofluoresence staining was applied in the first part of our study. The results in Figure 1 provided convincing evidence that a polyclonal antibody preparation was capable of reacting with $M$. ovipneumoniae bacterial cells, therefore showing the validity and rationality of CPS antibody. This polyclonal antibody can be further used either in immunoblotting or ELISA analysis to demonstrate its ability to recognize and neutralize CPS in cells. 


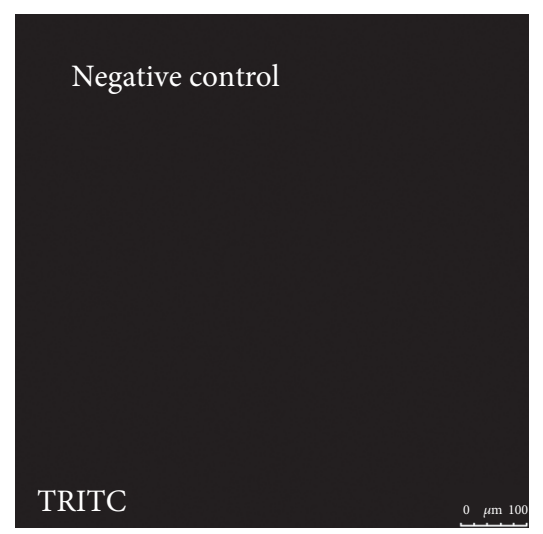

(a)

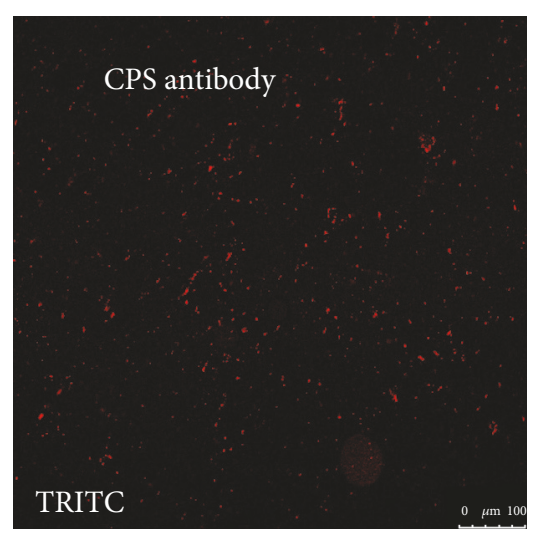

(c)

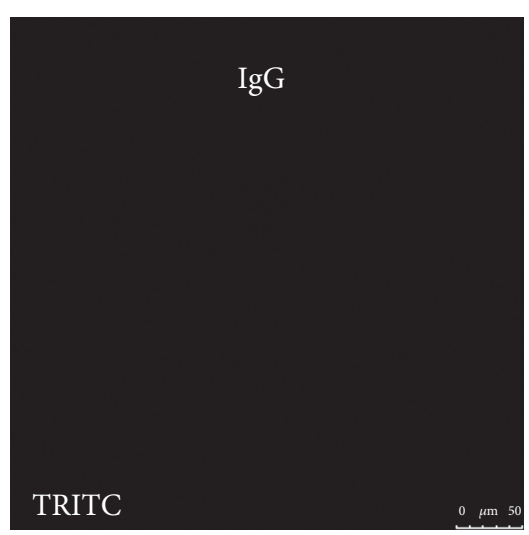

(e)

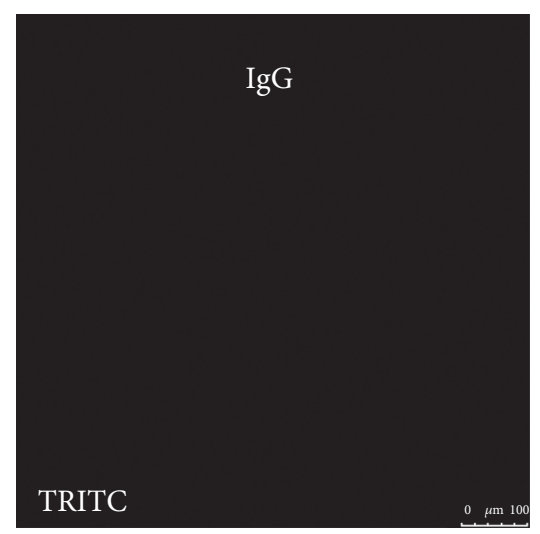

(b)

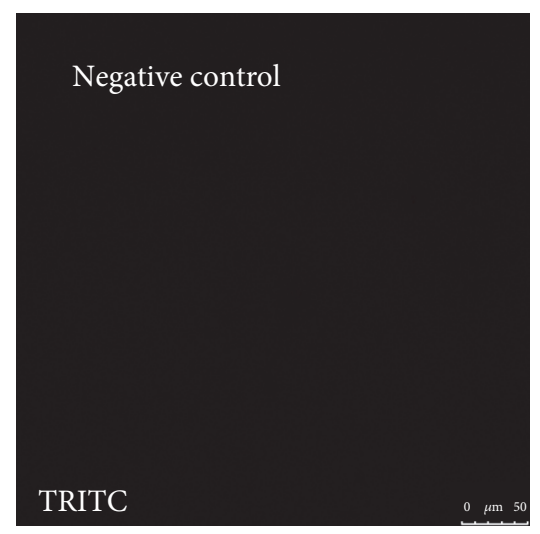

(d)

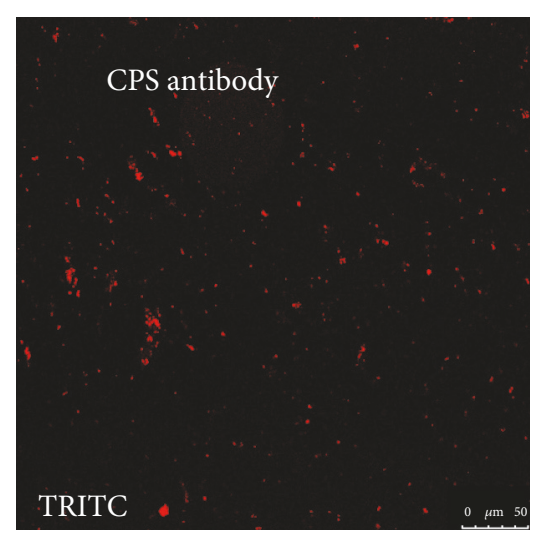

(f)

FIGURE 1: CPS antibody binds to M. ovipneumoniae in vitro. $1 \times 10^{10} \mathrm{CFU}$ (colony formation units) of M. ovipneumoniae solution were prepared, and the CFU value was adjusted by dilution before the test was conducted. For optimization of the immune reaction, $1 \times 10^{5}$ CFU were investigated. The bacterial cells were incubated with rabbit anti-M. ovipneumoniae CPS IgG ( $1: 100)$ and rabbit nonspecific IgG and then probed with Rhodamine- (TRITC-) labeled secondary antibody for red fluorescence. Representative images of confocal microscopy for M. ovipneumoniae after binding of CPS antibody indicate the presence of CPS (red) and confirm the validity of CPS antibody. Bars in (a)-(d): $100 \mu \mathrm{m}$.

3.2. CPS is a Key Component of M. Ovipneumoniae-Activated TLR Signaling in Sheep Bronchial Epithelial Cells. Considering that CPS generally plays a crucial role in the mechanism of specific recognition between host and pathogen interactions, we therefore sought to interrogate the impact of CPS treatment on TLR signaling activation in sheep bronchial epithelial cells. As expected, results showed transcripts of toll-like receptors (TLRs) including TLR1, TLR2, TLR4, and TLR6 which were significantly elevated upon CPS or M. ovipneumoniae stimulation (Figure 2(a)). Additionally, cells treated with CPS antibody exhibited a decreased ability to induce the expression of TLRs following M. ovipneumoniae infection (Figure 2(a)), suggesting an involvement of TLRs signaling in epithelial cells in response to CPS or $M$. 


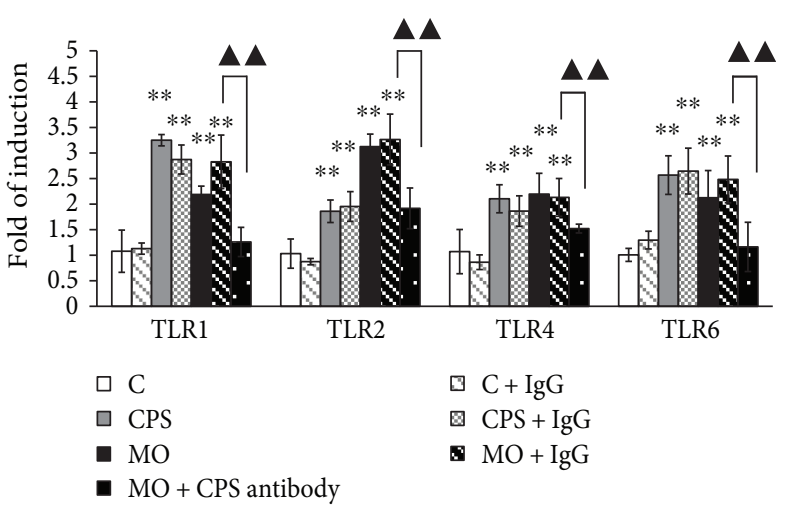

(a)

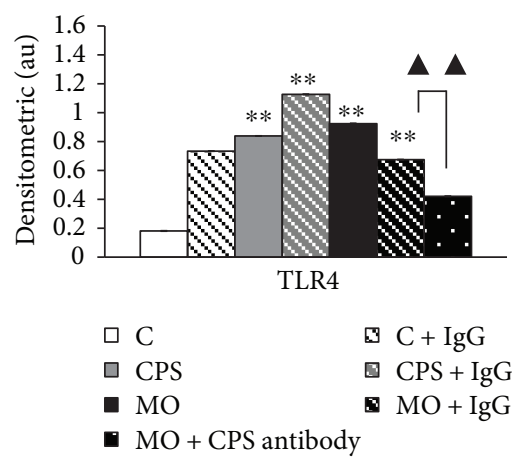

(c)

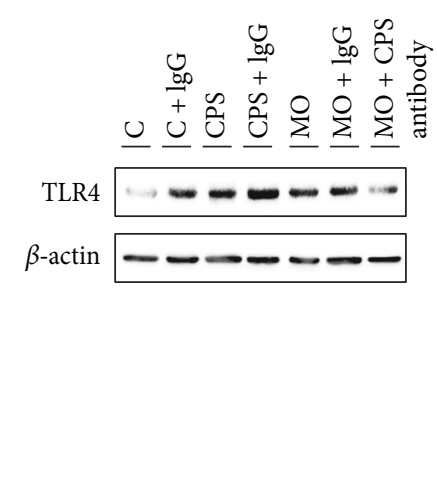

(b)

FIgURE 2: CPS stimulation induces the expression of TLRs in sheep bronchial epithelial cells. M. ovipneumoniae bacterial cells were preincubated with CPS antibody $(1: 100)$ at $4^{\circ} \mathrm{C}$ overnight. 4 -week old ALI cultures of sheep bronchial epithelial cells were then apically treated with capsular polysaccharide (CPS) at $100 \mathrm{ng} / \mathrm{mL}$ or infected with M. ovipneumoniae (MO) at MOI of 30 for $48 \mathrm{~h}$ before samples were harvested for analysis. For each treatment, an additional nonspecific IgG (1:100) was supplemented as a control. (a) The induction of TLR1, TLR2, TLR4, and TLR6 transcripts was determined by a qRT-PCR assay. All TLR transcripts showed a significant increase in the cells stimulated with CPS or M. ovipneumoniae, in comparison with the controls. The expression levels of these mRNA were calculated by $2^{-\Delta \Delta \mathrm{Ct}}$ method. (b) Immunoblotting assay also showed an evoked expression of TLR4 proteins in ALI cultures upon CPS/M. ovipneumoniae stimulation. (c) Densitometric values are shown for TLR4 proteins over $\beta$-actin. Values are mean \pm SD for at least three independent experiments performed in triplicate. ${ }^{* *} p<0.01$ versus that of the control. Compared between indicated groups, ${ }^{\mathbf{\Lambda}} p<0.01$.

ovipneumoniae stimulation. Consistently, the increased expression of TLR4 protein in response to an M. ovipneumoniae infection could be partially blocked by the addition of CPS antibody (Figures 2(b) and 2(c)).

3.3. The CPS Triggered a MyD88-Dependent TLR Signaling in Sheep Bronchial Epithelial Cells. To elucidate the underlying molecular mechanism of CPS activity, MyD88 and its downstream effectors, components of MyD88-dependent signaling pathway, were firstly accessed by western blot and/or qRT-PCR analysis. As shown in Figure 3, an evoked expression of MyD88, IRAK1, IRAK2, IRAK4, TRAF6, and TAB1 was detected following an exposure of CPS or M. ovipneumoniae at both of transcriptional (Figure 3(a)) and translational levels (Figures 3(b) and 3(c)) as compared with the uninfected control. Of note, a treatment of CPS antibody almost completely diminished the upregulated expression of MyD88-dependent signaling-associated proteins induced by an $M$. ovipneumoniae infection. These results imply that CPS plays a key role in M. ovipneumoniae-induced
MyD88-dependent TLR signaling activity in sheep airway epithelial cells.

3.4. The CPS Triggered a MyD88-Independent TLR Signaling in Sheep Bronchial Epithelial Cells. To further investigate whether the MyD88-independent signaling pathway was involved in CPS modulation of MO-induced proinflammatory responses in sheep epithelial cells, several key components of MyD88-independent signaling cascade, including TRAF3, TBK1, TRIF, and TRAM, were evaluated at both of transcriptional level (Figure 4(a)) and translational level (Figures 4(b) and 4(c)). An exposure to CPS or M. ovipneumoniae alone for $48 \mathrm{~h}$ resulted in a considerable elevation in the expression of TRAF3 transcript. In consistence, western blot analysis further confirmed an enhanced activation of TRAF3, TRIF, and TBK1 in the CPS- or M. ovipneumoniae-treated cells, despite the abundance of TBK1 transcript which was not remarkably increased. Additionally, a treatment of CPS antibody almost completely abolished the CPS- or M. ovipneumoniae-induced expression of above proteins, suggesting the M. ovipneumoniae-activated MyD88- 


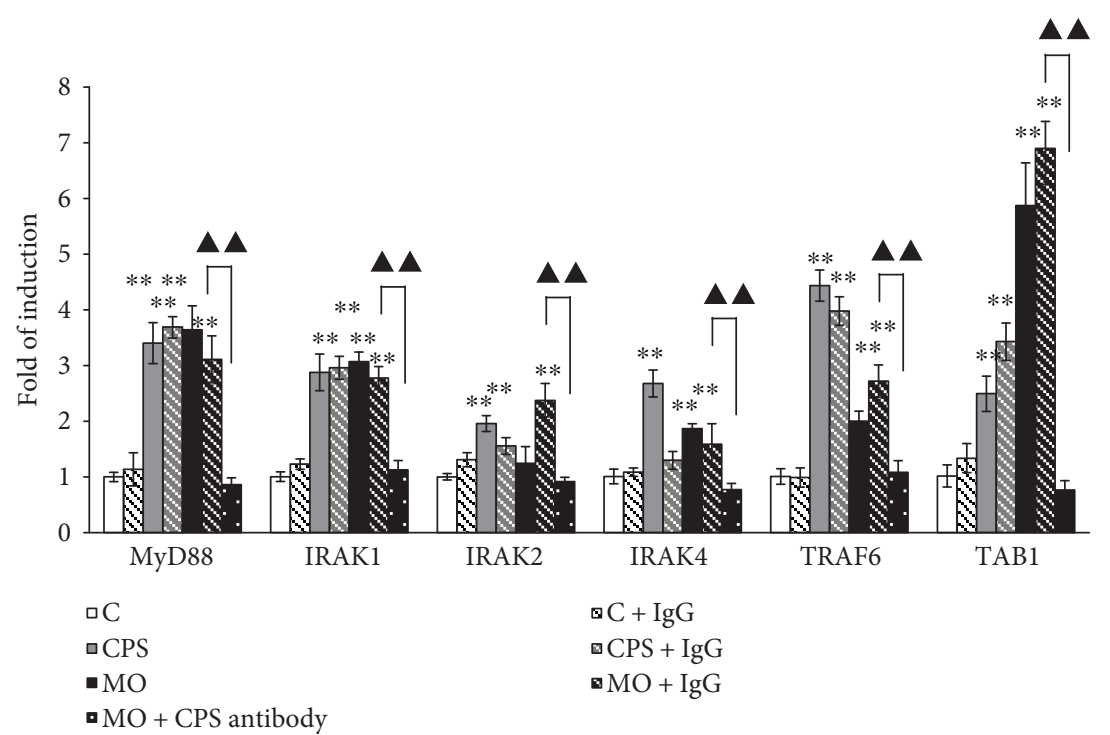

(a)

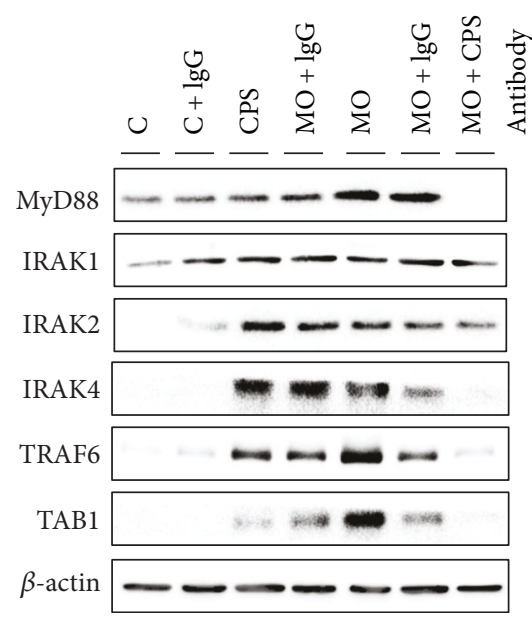

(b)

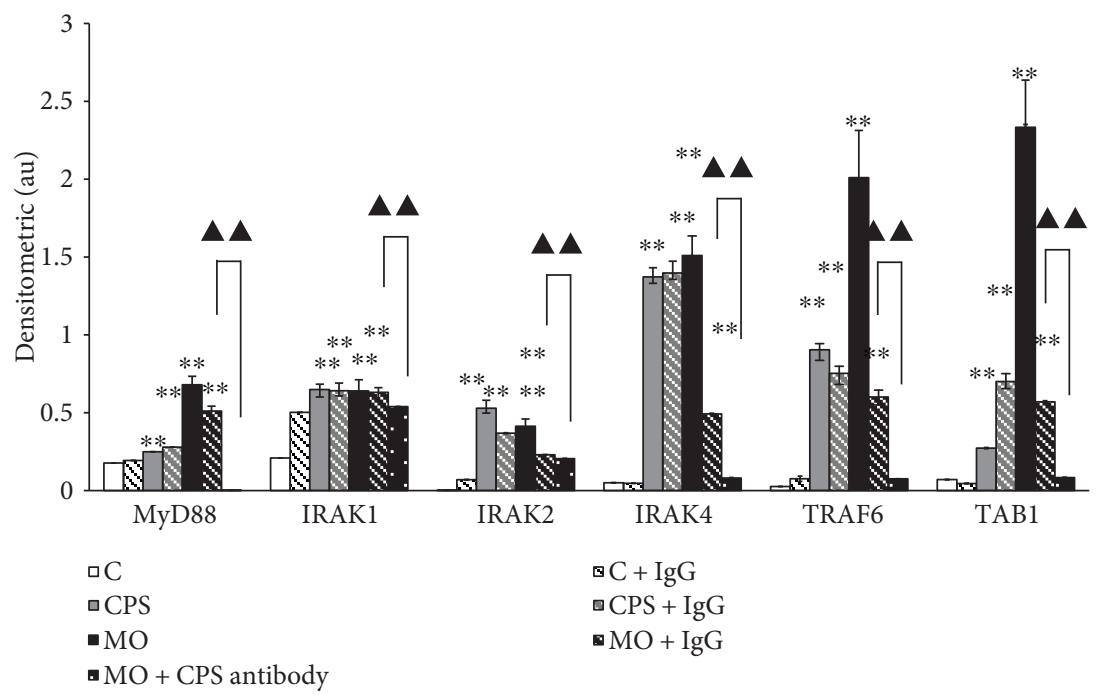

(c)

FIGURE 3: The impact of CPS on the expression of MyD88-dependent TLR signaling pathway in ALI cultures of sheep bronchial epithelial cells. 4-week old ALI cultures of sheep bronchial epithelial cells were exposed to indicated conditions for $48 \mathrm{~h}$. (a) The expression of several key components of MyD88-dependent signaling cascade, including the MyD88, IRAK1, IRAK2, IRAK4, TRAF6, and TAB1 transcripts were determined by qRT-PCR assays. In comparison with the controls, all tested transcripts showed a significant increase in the cells treated with CPS or M. ovipneumoniae. (b) Immunoblotting assay also showed an evoked expression of all MyD88-dependent signaling-associated proteins in ALI cultures upon CPS/MO. ovipneumoniae stimulation. (c) Densitometric analysis of western blot showed MyD88, IRAK1, IRAK2, IRAK4, TRAF6, and TAB1 expression over $\beta$-actin. Values are mean \pm SD for at least three independent experiments performed in triplicate. ${ }^{* *} p<0.01$ versus that of the control. Compared between indicated groups, ${ }^{\boldsymbol{\Lambda}} p<0.01$.

independent signaling pathway mainly attributed by CPS in sheep bronchial epithelial cells.

\subsection{The CPS Triggered an Activation of Nuclear Transcription} Factors in Sheep Bronchial Epithelial Cells. The occurrence of either TLRs interacting with MyD88 or TLR4 combining to the TRIF adaptors elicits downstream signaling events. Thus, we next assessed whether TLR-associated nuclear transcription factors were inducted and activated upon CPS or M. ovipneumoniae stimulation. Results showed that an exposure to CPS or M. ovipneumoniae alone evidently upregulated the expression of IRF3, IRF5, and NF- $\kappa \mathrm{B}$ as determined by a RT-PCR assay (Figure 5(a)) and/or an immunoblotting assay (Figures 5(b) and 5(c)). However, in the presence of CPS antibody, the protein of IRF3, as well as the phosphorylation of NF- $\kappa \mathrm{B}$ and $\mathrm{AP}-1$, only showed a baseline expression in response to a treatment of $M$. ovipneumoniae (Figures 5(b) and 5(c)). Of interest, no statistical difference was determined in the expression of IRF5 protein in CPS-treated cells compared with the uninfected control, 


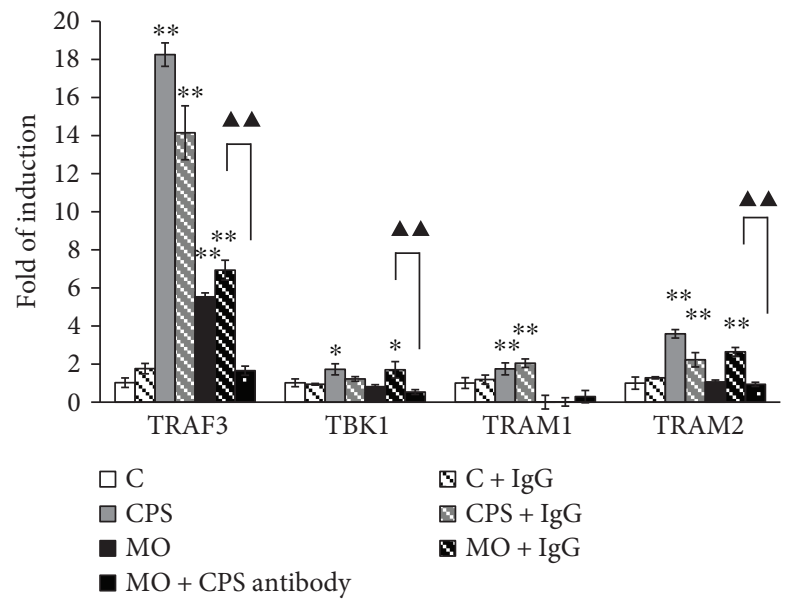

(a)

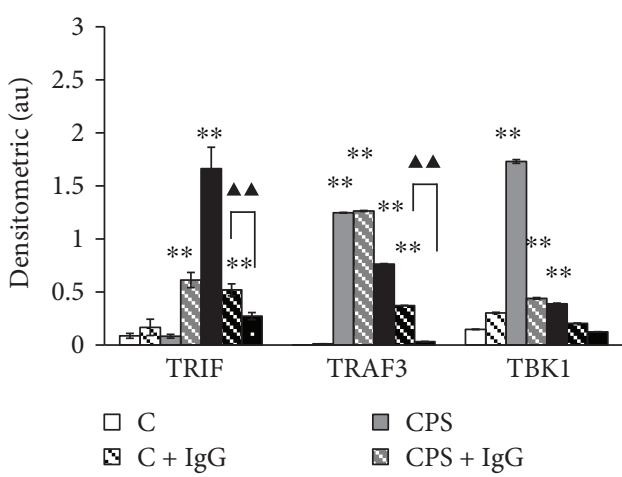

(b)

(c)

FIGURE 4: The impact of CPS on the expression of MyD88-independent TLR signaling pathway in ALI cultures of sheep bronchial epithelial cells. 4-week old ALI cultures of sheep bronchial epithelial cells were exposed to the indicated conditions for $48 \mathrm{~h}$. (a) The expression of several key components of MyD88-independent signaling cascade, including the TRAF3, TBK1, and TRAM transcripts were determined by qRTPCR assays. A significantly more abundant TRAF3 and TRAM2 mRNA was detected in ALI cultures treated with CPS/M. ovipneumoniae, in comparison with uninfected controls, while no evident alternations of TBK1 and TRAM1 expression were observed. (b) Expression levels of MyD88-independent signaling-associated proteins. Results showed that the expression of TRIF, TRAF3, and TBK1 were remarkably upregulated after treatments of CPS/M. ovipneumoniae. The immunoblots showed here were representative of three independent experiments with similar results. (c) Densitometric values are shown for TRIF, TRAF3, and TBK1 proteins over $\beta$-actin. Values are mean $\pm \mathrm{SD}$ for at least three independent experiments performed in triplicate. ${ }^{*} p<0.05$ and ${ }^{* *} p<0.01$ versus that of the control. Compared between indicated groups, ${ }^{\mathbf{\Lambda}} p<0.01$.

despite the abundance of its transcript which was evidently increased (Figure 5).

\subsection{The CPS Triggered the Production of Proinflammatory} Mediators and Cytokines in Sheep Bronchial Epithelial Cells. To further characterize the molecular mechanism of CPS activity, the concentrations of proinflammatory cytokines and chemokines were determined since they were major factors contributing to the initiation and amplification of inflammation. Relative mRNA expression data indicated that the expression of representative proinflammatory mediators including IL1 $\beta$, IL6, IL8, IL12, and TNF $\alpha$ were remarkably elevated in cells in response to CPS or M. ovipneumoniae treatment (Figures 6(a)-6(c)). Intriguingly, the expression of anti-inflammatory mediators such as IL10 and TGF- $\beta$ were also significantly induced following an exposure of CPS or M. ovipneumoniae alone (Figures 6(a)$6(\mathrm{c}))$. To confirm the findings from the qRT-PCR shown in Figures 6(a)-6(c), the release of $\mathrm{TNF} \alpha$, IL1 $\beta$, IL6, IL8, and IL10 in culture media of the upper chamber was further quantified by an ELISA assay. Results showed that the secretion of $\mathrm{TNF} \alpha, \operatorname{IL} 1 \beta$, IL8, and IL10 were synergistically increased upon CPS or $M$. ovipneumoniae stimulation (Figure 6(d)), therefore indicating a parallel impact between CPS and M. ovipneumoniae in the upregulation of proinflammatory gene expression. Additionally, CPS neutralization partially diminished cytokine accumulation and progression of M. ovipneumoniae-induced inflammation.

\section{Discussion}

Numerous studies have shown that inflammatory elements play a significant role in initiating and extending mycoplasma-associated diseases. However, the precise molecular mechanism following M. ovipneumoniae infections have not been explored and remain incompletely understood. In 


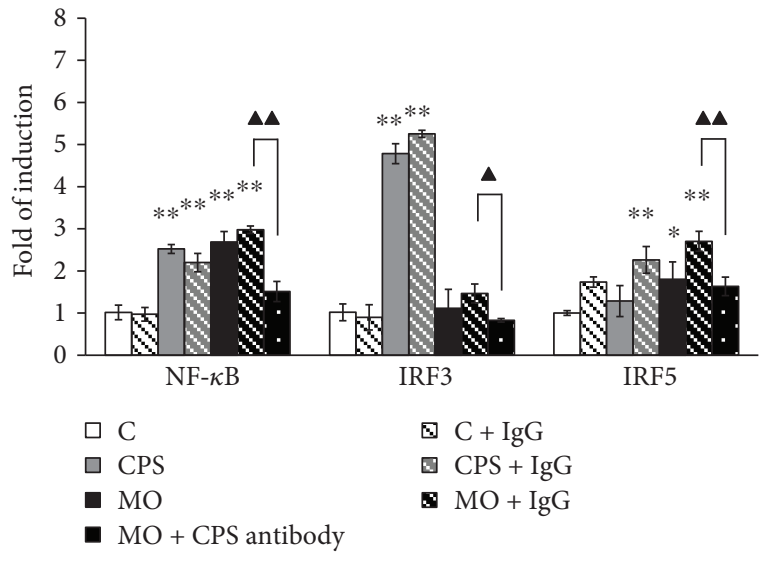

(a)

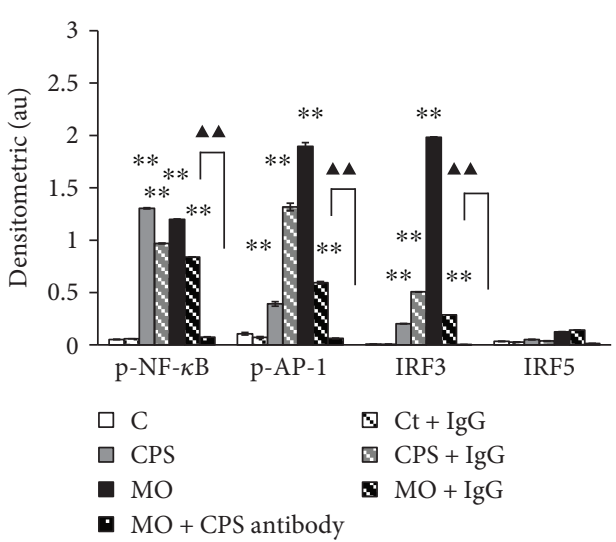

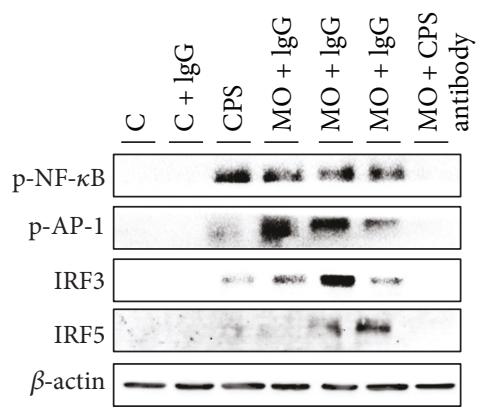

(b)

(c)

FIGURE 5: The impact of CPS on the expression of TLR signaling-associated nuclear transcription factors in ALI cultures of sheep bronchial epithelial cells. 4-week old ALI cultures of sheep bronchial epithelial cells were exposed to the indicated conditions for $48 \mathrm{~h}$. (a) The expression of several key nuclear transcription factors, including the IRF5, NF- $\kappa$ B, and IRF3 transcripts, were determined by qRT-PCR assays. An extremely more abundant IRF5 and NF- $\kappa$ B mRNA was detected in ALI cultures treated with CPS/MO, while no evident alternation of IRF3 expression were observed in M. ovipneumoniae-infected cells. (b) Immunoblotting assay also showed the expression of p-NF- $\kappa \mathrm{B}$, p-AP-1 and IRF3 protein, but not the IRF5 protein, was increased in ALI cultures upon CPS/M. ovipneumoniae stimulation. (c) Densitometric values are shown for $\mathrm{p}-\mathrm{NF}-\kappa \mathrm{B}$, p-AP-1, IRF3, and IRF5 proteins over $\beta$-actin. These bar graphs shown here are representative of three independent experiments with similar results. ${ }^{*} p<0.05$ and ${ }^{* *} p<0.01$ versus that of the control. Compared between indicated groups, ${ }^{\boldsymbol{\Delta}} p<0.05$ and ${ }^{\boldsymbol{\Lambda}} p<0.01$.

the present study, we identified for the first time that CPS is a key virulent component of M. ovipneumoniae that induces a TLR signaling-mediated inflammatory response in sheep bronchial cells, in which both CPS of M. ovipneumoniae and M. pneumoniae cells were able to trigger TLR4-MyD88-NF$\kappa \mathrm{B}$ and TLR4-TRIF-IRF3 signaling activations and lead the production of varied proinflammatory cytokines, suggesting a close-knit involvement of CPS in the pathogenesis of M. ovipneumoniae.

Inflammation is a complex host defensive response against various harmful stimuli such as injury, radiation, and pathogens $[34,35]$. The mechanism that underlies such phenomenon may be reflected in the context of activation of immune cells including monocytes and macrophages, leading to a secretion of various inflammatory mediators such as cytokines, chemokines, and subsequent nuclear signaling transcription factor [36, 37]. A baseline expression of cytokines and chemokines is supposed to be possibly involved in a sequence of immune-mediated processes, leading to the protection of host organism against pathogen invasion. However, increasing evidence has indicated an uncontrolled inflammatory response which is the main factor responsible for inflammatory diseases [37]. In most cases, polysaccharides cannot enter cells in a direct way due to the large molecular mass [38]. Emerging evidences have supported that the first step for polysaccharides to exert their functions is the recognition and identification of host cells by pattern recognition receptors (PRRs) $[38,39]$. In this context, TLRs, a family of 10 identified members in sheep that are evolutionarily conserved, broadly expressed receptors which can recognize exogenous pathogen-associated molecular patterns (PAMPs) and allow the host to detect microbial infection [40].

The roles of TLRs in the initiation and activation of host immune response have been extensively investigated upon mycoplasma infection. TLR2 was the first TLR family 


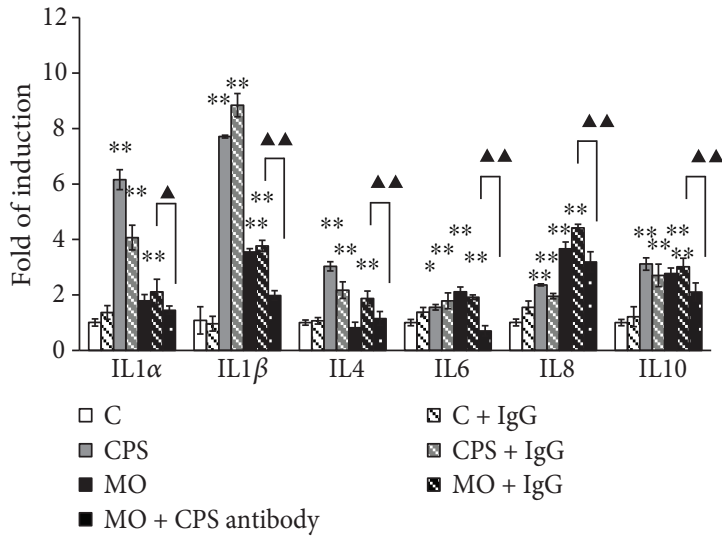

(a)

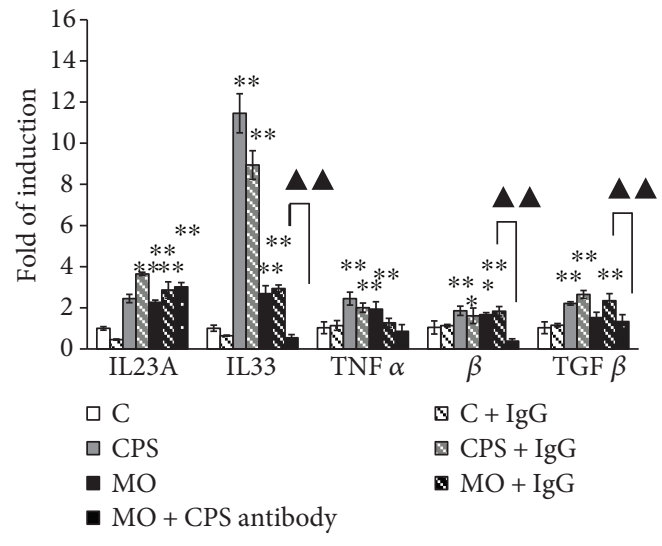

(c)

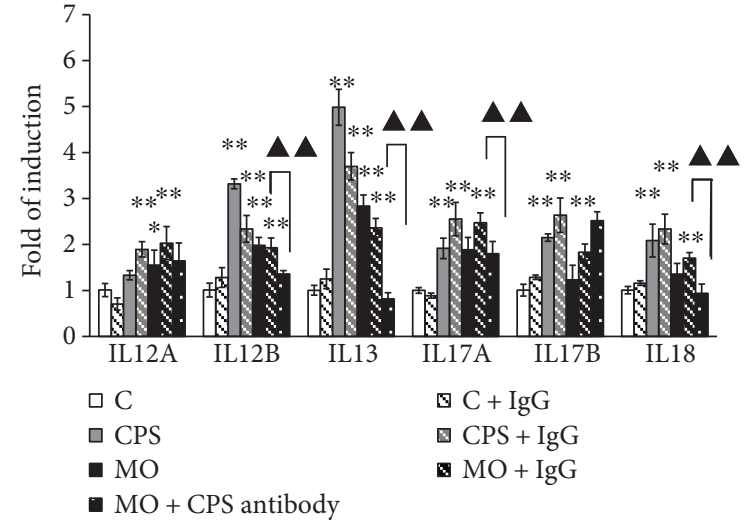

(b)

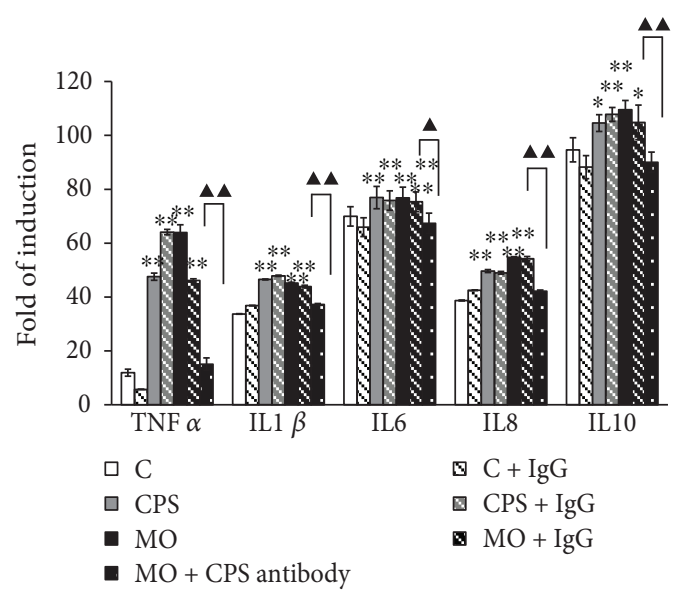

(d)

FIGURE 6: Induction of proinflammatory mediators and cytokines in the ALI cultures of sheep bronchial epithelial cells upon CPS stimulation. 4-week old ALI cultures of sheep bronchial epithelial cells were exposed to the indicated conditions for $48 \mathrm{~h}$ before the samples were harvested for analysis. (a-c) The mRNA expression of cytokines and chemokines including IL1 $\alpha$, IL1 $\beta$, IL4, IL6, IL8, IL10, IL12A, IL12B, IL13, IL17A, IL17B, IL18, IL23A, IL33, TNF $\alpha, \operatorname{IFN} \beta$, and TGF $\beta$ were determined by qRT-PCR assays. Compared to the control, all tested transcripts showed a significant increase after a treatment of CPS or M. ovipneumoniae. (d) Effect of CPS or MO on TNF $\alpha$, IL1 $\beta$, IL6, IL8, and IL10 production in the supernatant of ALI cultures were determined by ELISA. Results showed the protein expression of TNF $\alpha$, IL1 $\beta$, IL8, and IL10 was significantly elevated in cells upon CPS/M. ovipneumoniae stimulation, in comparison with uninfected controls, while no remarkable alteration of IL6 expression was observed. These bar graphs shown here are representative of three independent experiments with similar results. ${ }^{*} p<0.05$ and ${ }^{* *} p<0.01$ versus that of the control. Compared between indicated groups, ${ }^{\mathbf{\Delta}} p<0.05$ and ${ }^{\mathbf{\Delta}} p<0.01$.

member to be documented to response to endogenous ligands released during Mycoplasma pneumoniae infection, such as lipoproteins [41]. On account of the absence of a cell wall and inflammation-inducing endotoxin, lipoproteins were widely identified as an inflammation-associated factor during M. pneumoniae infection [42]. In general, TLR2 forms heterodimers either with TLR1 or TLR6 to attain specificity to recognize different triacylated and diacylated lipoproteins derived from M. pneumoniae [43]. Besides, TLR4, one major receptor for polysaccharide, plays a central role in coordinating the occurrence of innate immune response and accelerating the production of proinflammatory cytokines. Recent researches have indicated that $M$. pneumonia is capable of inducing inflammatory responses via a TLR2independent manner, in which TLR4 and autophagy are involved [42]. Herein, we tentatively explored the expression pattern of TLRs in ALI cultures of sheep epithelial cells in response to CPS or M. ovipneumoniae stimulation. Consistent with above findings, significant upregulation of TLR expression including TLR1, TLR2, TLR4, and TLR6 relative to the controls was observed in sheep epithelial cells following CPS or M. ovipneumoniae treatment. Of particular, antibody to CPS demonstrated an ability to block the activation of TLRs, of which anti-CPS antibodies to CPS showed a potential to block the binding of M. ovipneumoniae to epithelial cells in vitro, therefore preventing colonization and rapid multiplication of the pathogen. These results indicated that CPS is responsible for the recognition of M. ovipneumoniae by epithelial cells.

It is well known that TLR signaling is initiated upon the recognition of corresponding ligand, such as polysaccharide, by its intracellular toll/interleukin-1 receptor (TIR) domain, which commonly serves as a scaffold for protein-protein interaction, resulting in a downstream signaling cascade associated with inflammation [44, 45]. MyD88 is a key adaptor protein in the activation of TLR signaling pathway, 
required for all identified TLRs, with an exception of TLR3. Previous studies have described that MyD88-dependent pathway played an essential role in pulmonary inflammation by stimulating various cellular and molecular events, leading to exacerbation of lung damage. Initiation of MyD88 by TLRs first triggers the recruitment of IRAK family members in sequence, including IRAK4, IRAK1, and IRAK2 [46]. IRAK is released from the receptor and subsequently leads to the activation of TNF receptor-associated factor 6 (TRAF6), which eventually regulates gene transcription of inflammatory responses by activating downstream cytosolic proteins and nuclear transcription factors such as NF- $\kappa \mathrm{B}$ and AP-1 $[47,48]$. In agreement with this notion, our results showed that an exposure of CPS or an infection of M. ovipneumoniae significantly induced the upregulation of MyD88 and its downstream effectors including IRAKs, TRAF6 and TAB1, therefore indicating that a MyD88dependent pathway might be at least in part involved in the CPS- or M. ovipneumoniae-induced inflammation. Besides, antibody neutralization experiments showed that the antiCPS antibody effectively blocked M. ovipneumoniae-induced activation of MyD88-dependent signaling pathway from ALI cells, therefore suggesting that the CPS of this pathogen may be the main component responsible for inflammatory responses induced by $M$. ovipneumoniae infections.

Alternatively, it has been described that TLR stimulation can activate a MyD88-independent alternative pathway mediated by TIR-domain-containing adapter-inducing interferon- $\beta$ (TRIF) and IRF3. TRIF, one of the TIR domaincontaining adaptors, is a MyD88 homologous protein, which is specifically implicated in the TLR3- and TLR4-mediated MyD88-independent pathway [41]. Mechanistically, upon the activation, an array of downstream protein including TRAF3 and TBK1 is introduced. As the key transcription factor, IRF3 is then phosphorylated by TBK1 and is transferred into nucleus and bind with the ifn promoter, triggering ifn transcription [49]. Our results showed that the occurrence of inflammation induced by both CPS and M.ovipneumoniae was linked to a MyD88-independent signaling pathway due to the fact that TRIF and its associated downstream proteins were simultaneously upregulated in sheep epithelial cells following CPS and M. ovipneumoniae treatment. Meanwhile, the expression of $M$. ovipneumoniae-induced TRIF, TRAF3, TBK1, and TRAM expression was found to be markedly attenuated in the presence of CPS antibody, therefore indicating an important role of CPS in immune responses of $M$. ovipneumoniae during infections.

Given the fact of both MyD88-dependent and MyD88independent pathways were involved in the CPS of M. ovipneumoniae induced inflammation in ALI cultures of sheep bronchial epithelial cells, the underlying nuclear mechanisms by which CPS triggered inflammation were thus further explored. In the downstream of above signaling pathways, phosphorylation is pivotal for mediating nucleus translocation and functional transcription. Nuclear factor$\kappa \mathrm{B}(\mathrm{NF}-\kappa \mathrm{B})$ and activator protein-1 (AP-1) are two important downstream mediators of immediate early gene expression which couples extracellular signals to gene-activating events associated with a variety of inflammatory, apoptotic, and immune responses [50]. NF- $\kappa \mathrm{B}$ is normally comprised of a $50 \mathrm{kDa}$ subunit (p50) and a $65 \mathrm{kDa}$ subunit (p65) and is located within the cytoplasm in an inactive form via binding a member of the inhibitory kappa B $(\mathrm{I} \kappa \mathrm{B})$ family [51]. A characteristic feature of NF- $\kappa \mathrm{B}$ is its phosphorylation and translocation by posttranslational mechanisms related to the dissociation of $\mathrm{I} \kappa \mathrm{B}$ complex $[52,53]$. In addition, AP1 is a ubiquitous transcription factor that is mainly composed of the jun and fos gene products which form diverse homo (Jun/Jun) or heterodimeric (Jun/Fos) complex [54]. Once activated, the dimers are phosphorylated and translocated into the nucleus to bind target genes and subsequently initiate specific gene transcription [55]. As expected, our data showed that the activity of NF- $\kappa$ B and AP-1 was significantly increased in CPS or M. ovipneumoniae treated cells, which was partially inhibited by the treatment of CPS antibody. On the other hand, the activity of IRF3, a downstream nuclear transcription factor mediated by TRIF-dependent pathway, was also detected. In line with this hypothesis, an exposure of CPS or $M$. ovipneumoniae induces the increased expression of IRF3, thus initiating an immediate late gene transcription. To confirm the role of CPS in $M$. ovipneumoniae-activated signaling events, above key proteins were further analyzed in the presence of CPS antibody. As expected, the presence of anti-CPS antibodies led notably to the reduction of $M$. ovipneumoniae-induced phosphorylation of NF- $\kappa \mathrm{B}$ and AP-1, as well as the suppression of the nuclear translocations of IRF3 and IRF5. Taken as a whole, these results strongly suggest that both MyD88dependent and MyD88-independent signaling pathways play key roles in the response of sheep airway epithelial cells upon CPS or M. ovipneumoniae infection, especially CPS plays a key role in $M$. ovipneumoniae-activated TLR signaling in this cell type. However, further studies are needed to elucidate the relationship between MyD88-dependent and MyD88-independent pathways in order to uncover the precise mechanism underlying complex interactions in innate immune signaling.

Upon a stimulation of $M$. ovipneumoniae, inflammatory cytokines activate the immune system in response to "danger" and increase the efficiency of an immune response; the underlying mechanisms of immunomodulators are thought to occur via the activation of epithelial cells and macrophages, which secret an array of immune-associated factors, including TNF $\alpha$, IL1 $\beta$, IL6, IL8, and IL10, which are important in mediating both lung defense and inflammation [56]. Respectively, TNF $\alpha$ is implicated in acute phase of inflammation by activating epithelial cells and subsequent conducing to the generation of other inflammatory mediators [57]. As an important modular in the upper reaches of the immune response, IL1 $\beta$ participates in epithelial repair and amplifies the inflammatory cascade [16]. IL8 possesses neutrophil chemotactic and activating activities and is highly related to acute lung inflammatory injury. As a consequence, the study of cytokine and chemokine profiles may reveal the characteristics of innate immune mechanisms in epithelial cells following a mycoplasma infection. Indeed, a previous study has demonstrated that $M$. pneumoniae infection induces proinflammatory cytokine expression in human lung carcinoma 


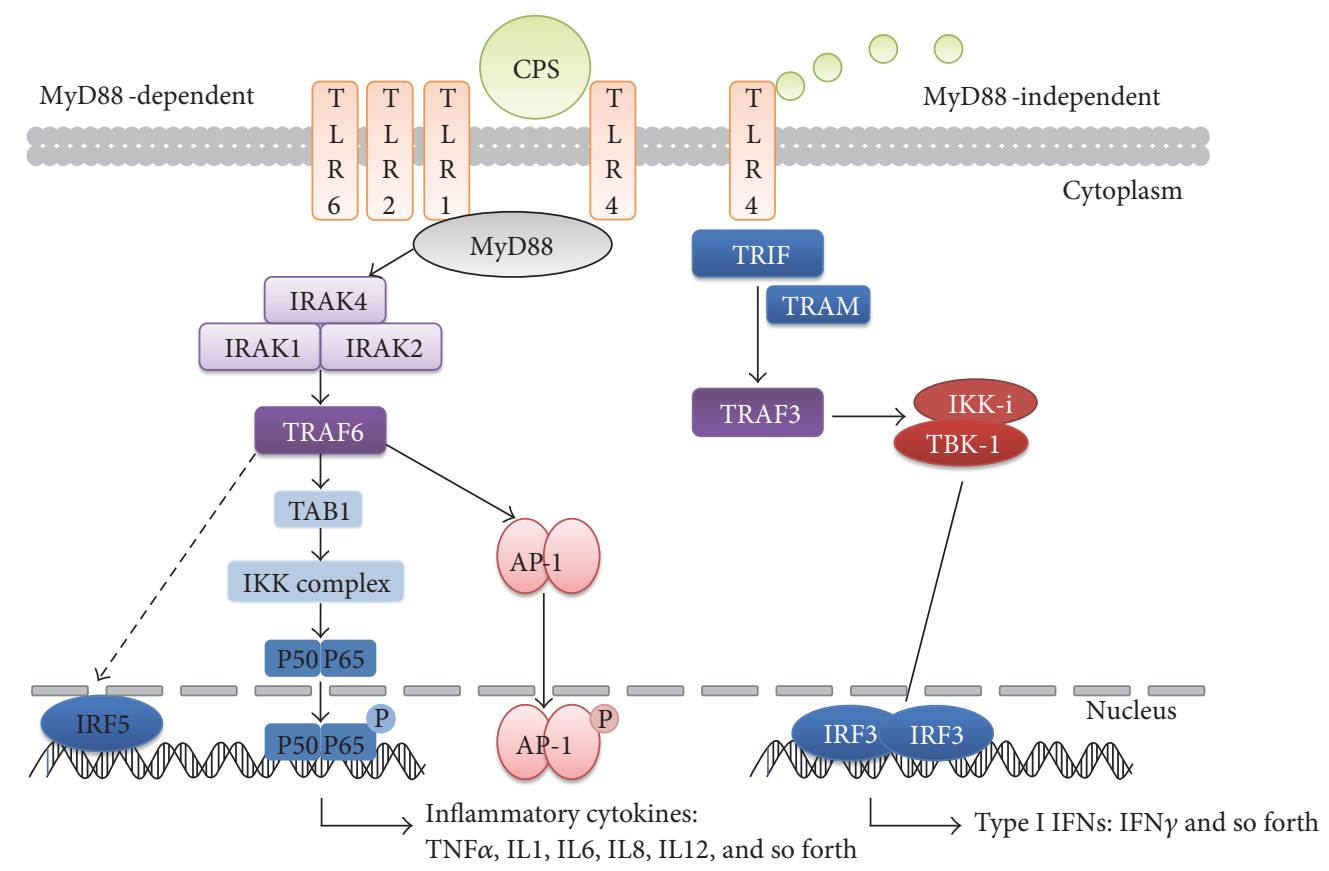

FIGURE 7: Scheme shows a possible mechanism of CPS-induced proinflammatory responses in ALI cultures of sheep bronchial epithelial cells. Both MyD88-dependent and MyD88-independent TLRs signaling pathways may play key roles in the sheep airway epithelial cells upon CPS or M. ovipneumoniae treatment.

cell line (A549) including IL1 $\beta$, IL6, IL8, and TNF $\alpha$ [58]. In addition, using human monocyte cell line (THP-1), Shimizu et al. have revealed a that wild-type stain of M. pneumoniae with cytoadherence ability is capable of inducing quite a number of proinflammatory cytokines such as TNF $\alpha$ and IL1 $\beta$ [59]. These data suggest that the proinflammation ability is involved in the pathogenesis of $M$. pneumoniae infection. Consistent with above conclusions, our results showed that the introduction of CPS or M. ovipneumoniae led to the upregulation of various inflammatory mediators, particularly including IL1 $\beta, \operatorname{TNF} \alpha$, and IL8, which are widely involved in the regulation of inflammation. To further identify the underlying immune mechanisms of CPS during $M$. ovipneumoniae-induced inflammation, a CPS antibody was used to continuously neutralize M. ovipneumoniae and assess the production of proinflammatory cytokines. Results showed that neutralization of CPS alleviated the M. ovipneumoniae-induced production of inflammatory mediators and effectors, therefore suggesting the close-knit involvement of CPS in M. ovipneumoniae-induced inflammation.

However, an excessive secretion of proinflammatory cytokines by immoderating immune response activation could subsequently trigger systemic inflammatory response and eventually contributing to the generation of amplification loops in the inflammatory cascade [60,61]. At present, imbalance between proinflammation and anti-inflammation is well known to play an essential role in the pathogenic process of pneumonia. It has been reported previously that $\operatorname{IL} 1 \beta$ is responsible for its own stimulation, thus resulting in an activation and amplification of IL-1R-mediated initiation of MyD88-dependent signaling pathways, eventually leading to the release of more proinflammatory cytokines including IL1 $\beta$ itself and hence an escalated host inflammatory response [13]. Besides, some other oxidants and inflammatory mediators such as TNF $\alpha$ are recorded to play a critical role in the inflammatory cascades as well [62], in which the dysregulation of TNF $\alpha$ is widely associated with the pathogenesis of several diseases, including septic shock, rheumatoid arthritis, and atherosclerosis [28, 63]. Therefore, the production of proinflammatory cytokines, such as IL1 $\beta$ and $\mathrm{TNF} \alpha$, is required to be strictly regulated in maintaining the balance between the proinflammatory and antiinflammatory responses. As anticipated, our results showed that the mRNA expression of IL10 and TGF $\beta$, two typical anti-inflammatory cytokines, were considerably enhanced following CPS or M. ovipneumoniae treatment. Noteworthy, the CPS-neutralized group showed lower levels of IL10 and TGF $\beta$ expression compared with the M. ovipneumoniae-treated group, therefore indicating CPS of M. ovipneumoniae could modulate the host immune function via releasing a mixture of both pro- and anti-inflammatory cytokines and chemokines from sheep epithelial cells.

Collectively, results from our study using an ALI culture of primary sheep bronchial epithelial cells suggest that CPS, which is the major capsular component of M. ovipneumoniae, might serve as main endogenous PAMPs of this pathogen to activate the epithelial inflammation. In this respect, CPS of $M$. ovipneumoniae could trigger inflammatory responses in sheep bronchial epithelial cells through a mechanism by which it activated both MyD88-dependent and MyD88independent signaling pathways and subsequently enhanced the production of various pro-inflammatory cytokines, such as IL1 $\beta$ and $\operatorname{TNF} \alpha$, and meanwhile increased the expression of anti-inflammatory cytokines, such as IL10 and TGF $\beta$ (Figure 7). This attribution of CPS makes it a promising vaccine candidate for $M$. ovipneumoniae infection as 
proinflammation may be an important step in the establishment of disease. Besides, considering inhibition of inflammatory mediators serves as a key strategy to control inflammation; specific agents that can suppress the transcription and translation of inflammation-associated genes may have a therapeutic potential for M. ovipneumoniae infections.

\section{Disclosure}

The funders had no role in the study design, data collection and analysis, decision to publish, or preparation of the manuscript.

\section{Conflicts of Interest}

The authors declare that they have no conflicts of interest.

\section{Authors' Contributions}

Xiaoming Liu and Yujiong Wang conceived and designed the experiments. Zhongjia Jiang, Fuyang Song, Yanan Li, Di Xue, Guangcun Deng, and Min Li performed the experiments and acquired the data. Zhongjia Jiang and Fuyang Song analyzed the data. Zhongjia Jiang drafted the manuscript. Yujiong Wang and Xiaoming Liu interpreted the data and critically revised the manuscript. All authors read and approved the final version of the manuscript.

\section{Acknowledgments}

This work was supported by grants from the National Natural Science Foundation of China (nos. 31460660 and 31572494).

\section{References}

[1] S. A. Broaders, W. C. Hooper, D. J. Phillips, and D. F. Talkington, "Mycoplasma pneumoniae subtype-independent induction of proinflammatory cytokines in THP-1 cells," Microbial Pathogenesis, vol. 40, no. 6, pp. 286-292, 2006.

[2] R. P. Dassanayake, S. Shanthalingam, C. N. Herndon et al., "Mycoplasma ovipneumoniae can predispose bighorn sheep to fatal Mannheimia haemolytica pneumonia," Veterinary Microbiology, vol. 145, no. 3-4, pp. 354-359, 2010.

[3] T. E. Besser, E. Frances Cassirer, M. A. Highland et al., "Bighorn sheep pneumonia: sorting out the cause of a polymicrobial disease," Preventive Veterinary Medicine, vol. 108, no. 2-3, pp. 85-93, 2013

[4] M. G. Gabridge, H. Gunderson, S. L. Schaeffer, and Y. D. Barden-Stahl, "Ciliated respiratory epithelial monolayers: new model for Mycoplasma pneumoniae infection," Infection and Immunity, vol. 21, no. 1, pp. 333-336, 1978.

[5] B. M. Buddle, M. Herceg, and D. H. Davies, "Experimental infection of sheep with Mycoplasma ovipneumoniae and Pasteurella haemolytica," Veterinary Microbiology, vol. 9, no. 6, pp. 543-548, 1984.

[6] Y. Wu, H. Qiu, Y. Zeng et al., "Mycoplasma genitalium lipoproteins induce human monocytic cell expression of proinflammatory cytokines and apoptosis by activating nuclear factor kappa B," Mediators of Inflammation, vol. 2008, Article ID 195427, p. 8, 2008.
[7] B. Garcia-Morante, J. Segales, S. Lopez-Soria et al., "Induction of mycoplasmal pneumonia in experimentally infected pigs by means of different inoculation routes," Veterinary Research, vol. 47, no. 1, p. 54, 2016.

[8] S. Messier and R. F. Ross, "Interactions of Mycoplasma hyopneumoniae membranes with porcine lymphocytes," American Journal of Veterinary Research, vol. 52, no. 9, pp. 1497-1502, 1991.

[9] J. P. Caruso and R. F. Ross, "Effects of Mycoplasma hyopneumoniae and Actinobacillus (Haemophilus) pleuropneumoniae infections on alveolar macrophage functions in swine," American Journal of Veterinary Research, vol. 51, no. 2, pp. 227-231, 1990.

[10] M. Niang, R. F. Rosenbusch, J. Lopez-Virella, and M. L. Kaeberle, "Expression of functions by normal sheep alveolar macrophages and their alteration by interaction with Mycoplasma ovipneumoniae," Veterinary Microbiology, vol. 58, no. 1, pp. 31-43, 1997.

[11] Y. C. Lin, R. J. Miles, R. A. Nicholas, D. P. Kelly, and A. P. Wood, "Isolation and immunological detection of Mycoplasma ovipneumoniae in sheep with atypical pneumonia, and lack of a role for Mycoplasma arginini," Research in Veterinary Science, vol. 84, no. 3, pp. 367-373, 2008.

[12] M. Niang, R. F. Rosenbusch, J. Lopez-Virella, and M. L. Kaeberle, "Differential serologic response to Mycoplasma ovipneumoniae and Mycoplasma arginini in lambs affected with chronic respiratory disease," Journal of Veterinary Diagnostic Investigation, vol. 11, no. 1, pp. 34-40, 1999.

[13] C. W. Brinson, Z. Lu, Y. Li, M. F. Lopes-Virella, and Y. Huang, "Lipopolysaccharide and IL-1beta coordinate a synergy on cytokine production by upregulating MyD88 expression in human gingival fibroblasts," Molecular Immunology, vol. 79, no. 1, pp. 47-54, 2016.

[14] M. Niang, R. F. Rosenbusch, J. J. Andrews, and M. L. Kaeberle, "Demonstration of a capsule on Mycoplasma ovipneumoniae," American Journal of Veterinary Research, vol. 59, no. 5, pp. 557-562, 1998.

[15] K. Brune, J. Frank, A. Schwingshackl, J. Finigan, and V. K. Sidhaye, "Pulmonary epithelial barrier function: some new players and mechanisms," American Journal of Physiology. Lung Cellular and Molecular Physiology, vol. 308, no. 8, pp. L731-L745, 2015.

[16] M. Wang, X. B. Yang, J. W. Zhao, C. J. Lu, and W. Zhu, "Structural characterization and macrophage immunomodulatory activity of a novel polysaccharide from Smilax glabra Roxb," Carbohydrate Polymers, vol. 156, pp. 390402, 2017.

[17] L. E. Comstock and D. L. Kasper, "Bacterial glycans: key mediators of diverse host immune responses," Cell, vol. 126, no. 5, pp. 847-850, 2006

[18] Z. Liu, X. You, Z. Peng et al., "Mycoplasma pneumoniae capsular polysaccharides bind to DC-SIGN and promote the secretion of IL-10," Xi Bao Yu fen Zi Mian Yi Xue Za Zhi, vol. 29, no. 1, pp. 10-13, 2013.

[19] R. P. Schleimer, A. Kato, R. Kern, D. Kuperman, and P. C. Avila, "Epithelium: at the interface of innate and adaptive immune responses," The Journal of Allergy and Clinical Immunology, vol. 120, no. 6, pp. 1279-1284, 2007.

[20] K. B. Waites and D. F. Talkington, "Mycoplasma pneumoniae and its role as a human pathogen," Clinical Microbiology Reviews, vol. 17, no. 4, pp. 697-728, 2004. 
[21] Y. Wang, Z. Zhu, T. D. Church et al., "SHP-1 as a critical regulator of Mycoplasma pneumoniae-induced inflammation in human asthmatic airway epithelial cells," Journal of Immunology, vol. 188, no. 7, pp. 3371-3381, 2012.

[22] R. K. Nelli, R. Maes, M. Kiupel, and G. S. Hussey, "Use of a feline respiratory epithelial cell culture system grown at the air-liquid interface to characterize the innate immune response following feline herpesvirus 1 infection," Virus Research, vol. 214, no. 1, pp. 39-48, 2016.

[23] W. Xu, A. J. Janocha, R. A. Leahy et al., "A novel method for pulmonary research: assessment of bioenergetic function at the air-liquid interface," Redox Biology, vol. 2, pp. 513519, 2014

[24] H. Matsui, S. H. Randell, S. W. Peretti, C. W. Davis, and R. C. Boucher, "Coordinated clearance of periciliary liquid and mucus from airway surfaces," The Journal of Clinical Investigation, vol. 102, no. 6, pp. 1125-1131, 1998.

[25] X. Liu, M. Luo, L. Zhang, W. Ding, Z. Yan, and J. F. Engelhardt, "Bioelectric properties of chloride channels in human, pig, ferret, and mouse airway epithelia," American Journal of Respiratory Cell and Molecular Biology, vol. 36, no. 3, pp. 313-323, 2007.

[26] Y. Ma, F. Han, J. Liang et al., “A species-specific activation of toll-like receptor signaling in bovine and sheep bronchial epithelial cells triggered by Mycobacterial infections," Molecular Immunology, vol. 71, no. 1, pp. 23-33, 2016.

[27] D. Xue, Y. Ma, M. Li et al., "Mycoplasma ovipneumoniae induces inflammatory response in sheep airway epithelial cells via a MyD88-dependent TLR signaling pathway," Veterinary Immunology and Immunopathology, vol. 163, no. 1-2, pp. 57-66, 2015.

[28] P. Z. Allen and B. Prescott, "Immunochemical studies on a Mycoplasma pneumoniae polysaccharide fraction: crossreactions with type 23 and 32 antipneumococcal rabbit sera," Infection and Immunity, vol. 20, no. 2, pp. 421-429, 1978.

[29] J. Wang, X. Bing, K. Yu, H. Tian, W. Wang, and S. Ru, "Preparation of a polyclonal antibody against goldfish (Carassius auratus) vitellogenin and its application to detect the estrogenic effects of monocrotophos pesticide," Ecotoxicology and Environmental Safety, vol. 111, no. 1, pp. 109-116, 2015.

[30] Y. Li, Z. Jiang, D. Xue et al., "Mycoplasma ovipneumoniae induces sheep airway epithelial cell apoptosis through an ERK signalling-mediated mitochondria pathway," $B M C$ Microbiology, vol. 16, no. 1, p. 222, 2016.

[31] X. Liu, Z. Yan, M. Luo, and J. F. Engelhardt, "Species-specific differences in mouse and human airway epithelial biology of recombinant adeno-associated virus transduction," American Journal of Respiratory Cell and Molecular Biology, vol. 34, no. 1, pp. 56-64, 2006.

[32] K. J. Livak and T. D. Schmittgen, "Analysis of relative gene expression data using real-time quantitative PCR and the 2(-Delta Delta C(T)) Method," Methods, vol. 25, no. 4, pp. 402-408, 2001.

[33] J. Liu, K. L. Ma, Y. Zhang et al., “Activation of mTORC1 disrupted LDL receptor pathway: a potential new mechanism for the progression of non-alcoholic fatty liver disease," The International Journal of Biochemistry \& Cell Biology, vol. 61, no. 1, pp. 8-19, 2015.

[34] Y. Diao, Y. Xin, Y. Zhou et al., "Extracellular polysaccharide from Bacillus sp. strain LBP32 prevents LPS-induced inflammation in RAW 264.7 macrophages by inhibiting NF-kappa
B and MAPKs activation and ROS production," International Immunopharmacology, vol. 18, no. 1, pp. 12-19, 2014.

[35] K. Tai, H. Iwasaki, S. Ikegaya, and T. Ueda, "Minocycline modulates cytokine and chemokine production in lipopolysaccharide-stimulated THP-1 monocytic cells by inhibiting Ikappa B kinase alpha/beta phosphorylation," Translational Research, vol. 161, no. 2, pp. 99-109, 2013.

[36] C. D. Munhoz, B. Garcia-Bueno, J. L. Madrigal, L. B. Lepsch, C. Scavone, and J. C. Leza, "Stress-induced neuroinflammation: mechanisms and new pharmacological targets," Brazilian Journal of Medical and Biological Research, vol. 41, no. 12, pp. 1037-1046, 2008.

[37] X. Zong, D. Song, T. Wang et al., "LFP-20, a porcine lactoferrin peptide, ameliorates LPS-induced inflammation via the MyD88/NF-kappa B and MyD88/MAPK signaling pathways," Developmental and Comparative Immunology, vol. 52, no. 2, pp. 123-131, 2015.

[38] C. Lull, H. J. Wichers, and H. F. Savelkoul, “Antiinflammatory and immunomodulating properties of fungal metabolites," Mediators of Inflammation, vol. 2005, no. 2, pp. 63-80, 2005.

[39] R. Medzhitov and C. Janeway Jr., "Innate immune recognition: mechanisms and pathways," Immunological Reviews, vol. 173, no. 1, pp. 89-97, 2000.

[40] P. G. Tipping, "Toll-like receptors: the interface between innate and adaptive immunity," Journal of the American Society of Nephrology, vol. 17, no. 7, pp. 1769-1771, 2006.

[41] K. Takeda and S. Akira, "TLR signaling pathways," Seminars in Immunology, vol. 16, no. 1, pp. 3-9, 2004.

[42] T. Shimizu, "Inflammation-inducing factors of Mycoplasma pneumoniae," Frontiers in Microbiology, vol. 7, p. 414, 2016.

[43] A. Ozinsky, D. M. Underhill, J. D. Fontenot et al., “The repertoire for pattern recognition of pathogens by the innate immune system is defined by cooperation between toll-like receptors," Proceedings of the National Academy of Sciences of the United States of America, vol. 97, no. 25, pp. 13766-13771, 2000.

[44] A. P. West, A. A. Koblansky, and S. Ghosh, "Recognition and signaling by toll-like receptors," Annual Review of Cell and Developmental Biology, vol. 22, pp. 409-437, 2006.

[45] S. Z. Xie, R. Hao, X. Q. Zha, L. H. Pan, J. Liu, and J. P. Luo, "Polysaccharide of Dendrobium huoshanense activates macrophages via toll-like receptor 4-mediated signaling pathways," Carbohydrate Polymers, vol. 146, pp. 292-300, 2016.

[46] M. Endale, S. C. Park, S. Kim et al., "Quercetin disrupts tyrosine-phosphorylated phosphatidylinositol 3-kinase and myeloid differentiation factor-88 association, and inhibits MAPK/AP-1 and IKK/NF-kappa B-induced inflammatory mediators production in RAW 264.7 cells," Immunobiology, vol. 218, no. 12, pp. 1452-1467, 2013.

[47] T. Kawai, O. Adachi, T. Ogawa, K. Takeda, and S. Akira, "Unresponsiveness of MyD88-deficient mice to endotoxin," Immunity, vol. 11, no. 1, pp. 115-122, 1999.

[48] W. Tian, C. Zhao, Q. Hu, J. Sun, and X. Peng, "Roles of toll-like receptors 2 and 6 in the inflammatory response to Mycoplasma gallisepticum infection in DF-1 cells and in chicken embryos," Developmental and Comparative Immunology, vol. 59, pp. 39-47, 2016.

[49] F. Sun, Y. B. Zhang, T. K. Liu, J. Shi, B. Wang, and J. F. Gui, "Fish MITA serves as a mediator for distinct fish IFN gene activation dependent on IRF3 or IRF7," Journal of Immunology, vol. 187, no. 5, pp. 2531-2539, 2011. 
[50] H. Schenk, M. Klein, W. Erdbrugger, W. Dröge, and K. Schulze-Osthoff, "Distinct effects of thioredoxin and antioxidants on the activation of transcription factors NF-kappa B and AP-1," Proceedings of the National Academy of Sciences of the United States of America, vol. 91, no. 5, pp. 1672-1676, 1994.

[51] S. Ghosh, M. J. May, and E. B. Kopp, "NF-kappa B and Rel proteins: evolutionarily conserved mediators of immune responses," Annual Review of Immunology, vol. 16, no. 1, pp. 225-260, 1998.

[52] T. Kawai and S. Akira, "Signaling to NF-kappa B by toll-like receptors," Trends in Molecular Medicine, vol. 13, no. 11, pp. 460-469, 2007.

[53] M. J. May and S. Ghosh, "Signal transduction through NFkappa B,” Immunology Today, vol. 19, no. 2, pp. 80-88, 1998.

[54] O. Uluckan, J. Guinea-Viniegra, M. Jimenez, and E. F. Wagner, "Signalling in inflammatory skin disease by AP-1 (Fos/Jun)," Clinical and Experimental Rheumatology, vol. 33, no. 4, Supplement 92, pp. S44-S49, 2015.

[55] H. B. Schonthaler, J. Guinea-Viniegra, and E. F. Wagner, "Targeting inflammation by modulating the Jun/AP-1 pathway," Annals of the Rheumatic Diseases, vol. 70, Supplement 1, pp. i109-i112, 2011.

[56] N. A. Eisele and D. M. Anderson, "Host defense and the airway epithelium: frontline responses that protect against bacterial invasion and pneumonia," Journal of Pathogens, vol. 2011, Article ID 249802, p. 16, 2011.

[57] T. Chen, R. Wang, W. Jiang et al., "Protective effect of astragaloside IV against paraquat-induced lung injury in mice by suppressing Rho signaling," Inflammation, vol. 39, no. 1, pp. 483492, 2016.

[58] J. Yang, W. C. Hooper, D. J. Phillips, and D. F. Talkington, "Regulation of proinflammatory cytokines in human lung epithelial cells infected with Mycoplasma pneumoniae," Infection and Immunity, vol. 70, no. 7, pp. 3649-3655, 2002.

[59] T. Shimizu, Y. Kida, and K. Kuwano, "Cytoadherence-dependent induction of inflammatory responses by Mycoplasma pneumoniae," Immunology, vol. 133, no. 1, pp. 51-61, 2011.

[60] J. Qi and S. M. Kim, "Characterization and immunomodulatory activities of polysaccharides extracted from green alga Chlorella ellipsoidea," International Journal of Biological Macromolecules, vol. 95, pp. 106-114, 2016.

[61] J. Choi, H. J. Jung, K. T. Lee, and H. J. Park, “Antinociceptive and anti-inflammatory effects of the saponin and sapogenins obtained from the stem of Akebia quinata," Journal of Medicinal Food, vol. 8, no. 1, pp. 78-85, 2005.

[62] F. Yang, W. J. de Villiers, C. J. McClain, and G. W. Varilek, "Green tea polyphenols block endotoxin-induced tumor necrosis factor-production and lethality in a murine model," The Journal of Nutrition, vol. 128, no. 12, pp. 2334-2340, 1998.

[63] K. S. Ahn and B. B. Aggarwal, "Transcription factor NF-kappa B: a sensor for smoke and stress signals," Annals of the new York Academy of Sciences, vol. 1056, no. 1, pp. 218-233, 2005. 


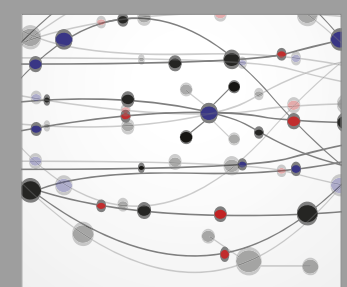

The Scientific World Journal
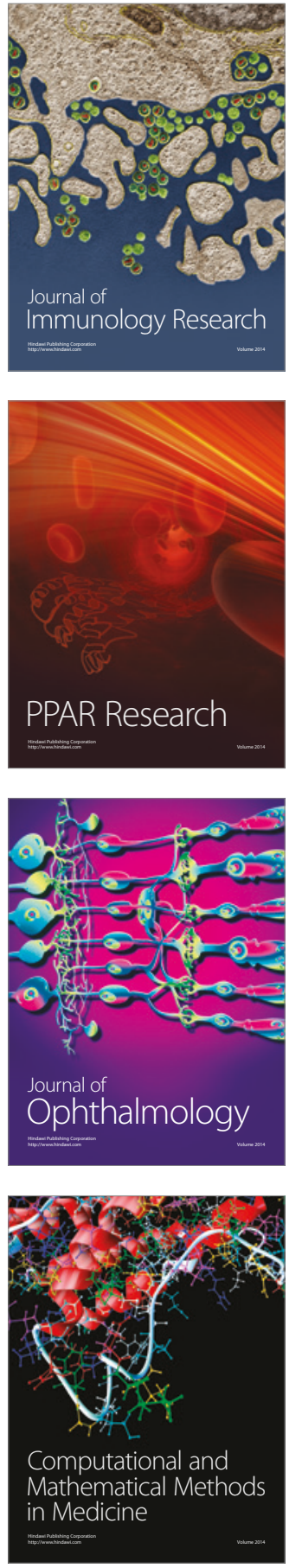

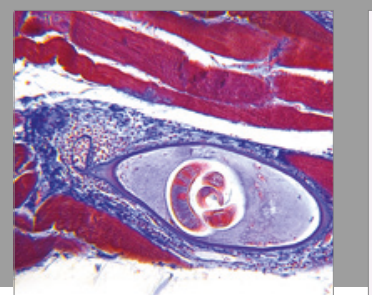

Gastroenterology Research and Practice
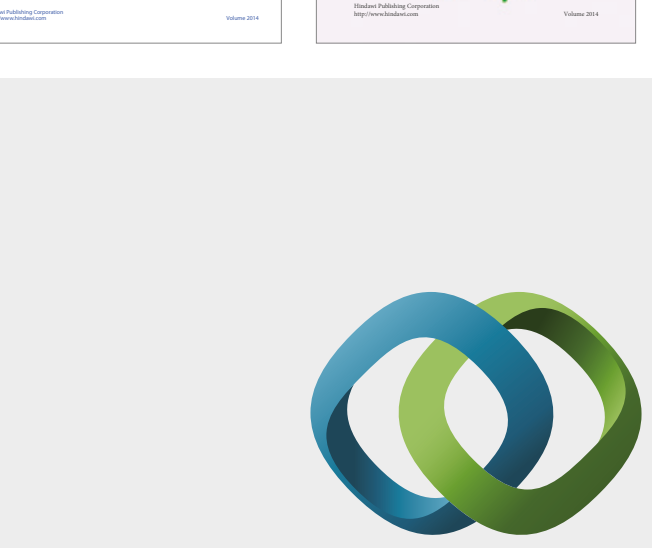

\section{Hindawi}

Submit your manuscripts at

https://www.hindawi.com
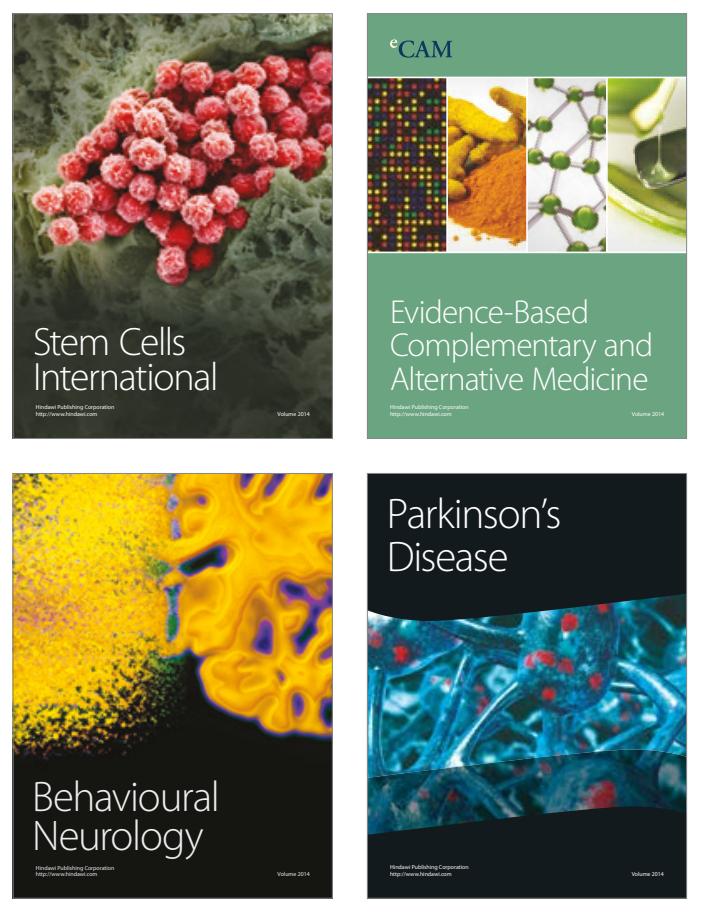
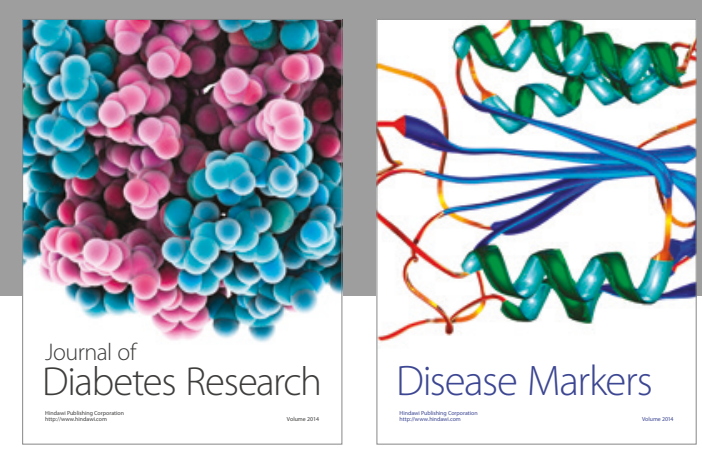

Disease Markers
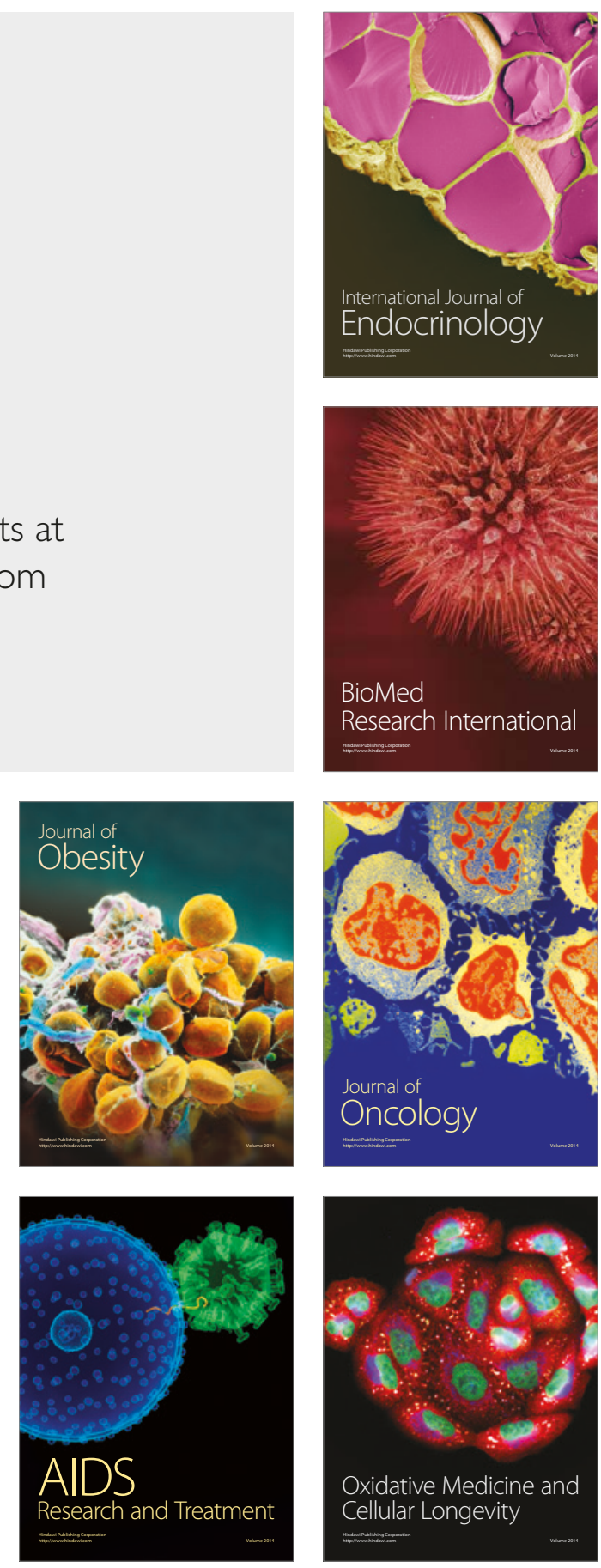